\title{
Minibrain/Dyrk1a Regulates Food Intake through the Sir2-FOXO-sNPF/NPY Pathway in Drosophila and Mammals
}

\author{
Seung-Hyun Hong ${ }^{19}$, Kyu-Sun Lee ${ }^{1,29}$, Su-Jin Kwak', Ae-Kyeong Kim ${ }^{1}$, Hua Bai ${ }^{3}$, Min-Su Jung ${ }^{4}$, \\ O-Yu Kwon ${ }^{5}$, Woo-Joo Song ${ }^{4}$, Marc Tatar ${ }^{3}$, Kweon $\mathbf{Y u}^{1,2 *}$
}

1 Aging Research Centre, Korea Research Institute of Bioscience and Biotechnology (KRIBB), Daejeon, Korea, 2 Functional Genomics Program, University of Science and Technology (UST), Daejeon, Korea, 3 Department of Ecology and Evolutionary Biology, Brown University, Providence, Rhode Island, United States of America, 4 Institute for Brain Science and Technology, FIRST Research Group, Inje University, Busan, Korea, 5 Department of Anatomy, School of Medicine, Chungnam National University, Daejeon, Korea

\begin{abstract}
Feeding behavior is one of the most essential activities in animals, which is tightly regulated by neuroendocrine factors. Drosophila melanogaster short neuropeptide F (sNPF) and the mammalian functional homolog neuropeptide Y (NPY) regulate food intake. Understanding the molecular mechanism of sNPF and NPY signaling is critical to elucidate feeding regulation. Here, we found that minibrain $(m n b)$ and the mammalian ortholog Dyrk1a target genes of sNPF and NPY signaling and regulate food intake in Drosophila melanogaster and mice. In Drosophila melanogaster neuronal cells and mouse hypothalamic cells, sNPF and NPY modulated the mnb and Dyrk1a expression through the PKA-CREB pathway. Increased Dyrk1a activated Sirt1 to regulate the deacetylation of FOXO, which potentiated FOXO-induced $s N P F / N P Y$ expression and in turn promoted food intake. Conversely, AKT-mediated insulin signaling suppressed FOXO-mediated sNPF/ $N P Y$ expression, which resulted in decreasing food intake. Furthermore, human Dyrk1a transgenic mice exhibited decreased FOXO acetylation and increased NPY expression in the hypothalamus, as well as increased food intake. Our findings demonstrate that Mnb/Dyrk1a regulates food intake through the evolutionary conserved Sir2-FOXO-sNPF/NPY pathway in Drosophila melanogaster and mammals.
\end{abstract}

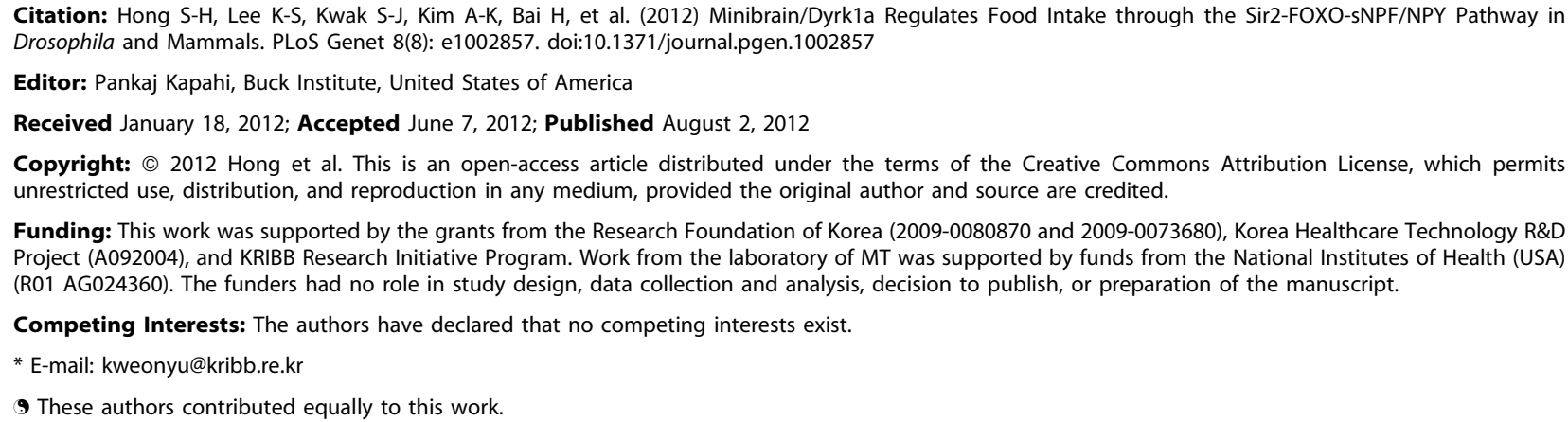

Funding: This work was supported by the grants from the Research Foundation of Korea (2009-0080870 and 2009-0073680), Korea Healthcare Technology R\&D Project (A092004), and KRIBB Research Initiative Program. Work from the laboratory of MT was supported by funds from the National Institutes of Health (USA) (R01 AG024360). The funders had no role in study design, data collection and analysis, decision to publish, or preparation of the manuscript.

Competing Interests: The authors have declared that no competing interests exist.

* E-mail: kweonyu@kribb.re.kr

9 These authors contributed equally to this work.

\section{Introduction}

Neuropeptides regulate a wide range of physiological processes in animals. In mammals, NPY is widely distributed in the brain and involved in various physiological functions including food intake. In the mammalian brain, the hypothalamus is the center for controlling food intake. The hypothalamic injection of NPY in the rat brain induces hyperphagia and obesity. In the hypothalamus, the arcuate nucleus (ARC) that contains orexigenic NPY and AgRP expressing neurons and anorexigenic POMC neurons senses hormonal levels of insulin and leptin and regulates food intake [1]. In Drosophila, sNPF, a functional homolog of NPY produced in sNPFnergic neurons of the fly brain, regulates food intake and growth [2]. Recently, we reported that sNPF and sNPF receptor (sNPFR1) regulate body growth through evolutionary conserved ERK-mediated insulin signaling in Drosophila and rat insulinoma cells [3].

Drosophila Minibrain (Mnb) and its mammalian ortholog Dual specificity tyrosine-phosphorylation-regulated kinase la (Dyrkla) are highly expressed in the neural tissues $[4,5,6]$. The Dyrkla gene has been implicated in Down Syndrome (DS) [5,7] and the expression level of Dyrk1a is increased in DS patients and Ts65Dn mice, a mouse model of Down syndrome [4,8]. Mutations of mnb and Dyrk1a in Drosophila and mammals show neural phenotypes like defects in neuroblasts proliferation and brain development $[6,9]$. Human patients with truncated mutations in the Dyrkla gene also show microcephaly [10,11]. To date, however, the effects of $m n b$ and Dyrkla upon food intake have not been described.

FoxOl modulates food intake by regulation of orexigenic Argp and anorexigenic Pomc genes in the hypothalamus of mice. In the ARC of hypothalamic neurons, FoxOl is localized in the nuclei during fasting and in the cytoplasm by feeding [12]. Sirtuin 1 (Sirt1), the mammalian ortholog of Drosophila Silent information regulator 2 (Sir2), in the ARG also regulates food intake [13]. The Sirtl protein level increases during fasting. Sirt 1 inhibition by the hypothalamic knock-out in the AgRP neurons decreases food intake [14]. In N43 hypothalamic cells, pharmacological inhibition of Sirtl increases anorexigenic POMC expression but co-treatment with Sirtl inhibitor and FoxOl siRNA does not [15], suggesting 


\section{Author Summary}

Feeding behavior is one of the most essential activities in animals. Abnormal feeding behaviors cause metabolic syndromes including obesity and diabetes. Neuropeptides regulate feeding behavior in animals from nematode to human. Here, we presented molecular genetic evidences of how neuropeptides regulate food intake using fruit fly and mouse model systems. Drosophila short neuropetide F (sNPF) and the mammalian functional homolog neuropeptide $Y$ (NPY) are produced from neurons in the brain of fruit fly and mouse, respectively. These neuropeptides turned on the minibrain, in mammals also called Dyrk1a, a target gene through the PKA-CREB pathway. Then, this Mnb/Dyrk1a enzyme activated Sir2/Sirt1 enzyme, which activated FOXO transcriptional factor, turning on the expression of a sNPF/NPY target gene. The increased sNPF/NPY increased food intake in fruit flies and mice. On the contrary, increased food intake induced insulin and activated insulin signaling. When insulin signaling is activated, FOXO transcriptional factor inhibited expression of a $s N P F / N P Y$ target gene. The inhibited sNPF/NPY reduced food intake. These findings indicate that FOXO transcription factor acts as a gatekeeper for fastingfeeding transition by regulating sNPF/NPY expression in Drosophila and mammals.

that Sirtl-mediated FoxOl deactylation is involved in the regulation of $P O M C \mathrm{mRNA}$ and food intake.

In this study, we identified mnb and Dyrkla as target genes of sNPF and NPY signaling, respectively, and describe a molecular mechanism of how Mnb and Dyrkla regulate food intake in Drosophila and mice.

\section{Results}

sNPF Targets mnb to Regulate Food Intake in Drosophila

To find genes affected by sNPF signaling, we performed a DNA microarray analysis using the Affymetrix Drosophila Genome 2.0 Array GeneChip with mRNA extracted from Drosophila neuronal BG2-c6 cells treated with sNPF peptide. Among the 159 genes with at least a two-fold change, mRNA of mnb increased 34-fold compared to the control (Table S1). To test whether the expression of $m n b$ is dependent on sNPF signaling in vivo, we examined the expression levels of $m n b$ in $s \mathcal{N P F}$ and $s \mathcal{N P F R} 1$ mutants. When $s \mathcal{N} P F$ was overexpressed in sNPFnergic neurons with the $s \mathcal{N} P F-G a l 4$ driver [16] $\left(s \mathcal{N} P F>{ }_{s} \mathcal{N} P F, s \mathcal{N} P F>2 X s \mathcal{N} P F\right), m n b$

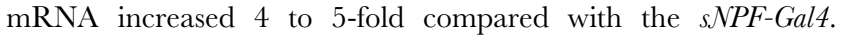
mRNA of $m n b$ decreased by less than half when $s N P F$ was inhibited $\left(s \mathcal{N} P F>{ }_{s} \mathcal{N} P F-R i\right)$ or by an $s \mathcal{N} P F$ mutant $\left(s \mathcal{N} P F^{c 00448}\right.$ ) (Figure $1 \mathrm{~A}$ and Figure S1A). When sNPFR1 was overexpressed via a sNPFR1Gal4 driver (Figure S2) (sNPFR1>sNPFR1), mnb mRNA was increased 3-fold compared with the sNPFR1-Gal4 control. When $s$ NPFR1 was inhibited ( $S N P F R 1>S N P F R 1-R i)$ or suppressed ( $N$ NPFR1>sNPFR1-DN), mnb mRNA was decreased by more than $50 \%$ (Figure $1 \mathrm{~A}$ and Figure S1A). Like mnb mRNA, Mnb proteins were also increased in $S \mathcal{N P F}$ or $s \mathcal{N P F R 1}$ overexpression with the $s \mathcal{N P F - G a l 4}$ or sNPFR1-Gal4 driver, (sNPF>2XsNPF, $s \mathcal{N} P F R 1>s \mathcal{N} P F R 1)$ while reduced in an $s \mathcal{N} P F$ mutant $\left(s \mathcal{N} P F^{000448}\right)$ or $s$ NPFR1 inhibition ( $s$ NPFR1> $N$ NPFR1-Ri) compared with the sNPF-Gal4 or sNPFR1-Gal4 control (Figure S3A). However, the numbers of Mnb expression neurons (asterisks) are consistent in the sNPFR1-Gal4 control, sNPFR1 overexpression

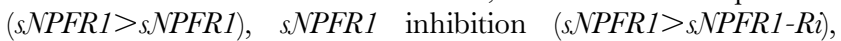
and an $s N P F^{00448}$ mutant (Figure S3B-S3F). These results indicate that sNPF-sNPFRl signaling regulates mnb mRNA and protein expression in Drosophila.

To understand how Mnb protein may interact with the sNPFR 1 receptor, we immunostained fly adult brains with Mnb and sNPFR1 antibodies. The Mnb antibody produced strong and weak staining in neuronal cells (Figure $1 \mathrm{H}, 1 \mathrm{~K}$, red) while the sNPFR 1 receptor antibody stained many neurons (Figure 1I, 1L, green). Among the strongly stained Mnb neurons, cell bodies of symmetrically localized median neurons behind the antennal lobe show overlap with the antibody against sNPFR1 (Figure 1J, 1M, arrows). At least ten neuronal cell bodies in median neurons were stained with the both antibodies. This coincidence suggests that at least part of Mnb function may be regulated by sNPF-sNPFR1 signaling.

Since sNPF signaling regulates food intake and growth, and growth is regulated by ERK-mediated insulin signaling [3], we hypothesized that sNPF may regulate food intake through the $m n b$ gene. To assess this hypothesis, we used the CAFÉ assay [17] to measure feeding in mnb mutant adults. Because homozygous mnb deletion mutants $\left(m n b^{d 305}\right.$ and $\left.m n b^{d 419}\right)$ generated by the imprecise excisions of the P-element (Figure S4A) are lethal (as are homozygous Dyrk1 a mutant mice) we analyzed mnb overexpression and hypomorphs generated by RNAi. mnb overexpression in sNPFR 1 neurons (sNPFR1>mnb) increased cumulative food consumption compared to the sNPFR1-Gal4 control whereas inhibiting mnb (sNPFR1>mnb-Ri) decreased cumulative food consumption (Figure 1C), indicating that $m n b$ expression in sNPFR 1 neurons can regulate food intake. Likewise, we measured the amount of food intake by the amount of digested dye from colored food. Overexpression of mnb in sNPFR1 neurons $(s \mathcal{N P F R} 1>m n b$ and $s \mathcal{N} P F R 1>2 \mathrm{Xmnb})$ increased consumed dye up to $57 \%$ compared with that of the $s$ NPFR1-Gal4 control whereas $m n b$ inhibition (sNPFR1>mnb-Ri) or the mnb mutant (mnb $\left.{ }^{G 1767}\right)$ decreased this intake by $30 \%$ (Figure $1 \mathrm{~B}$ and Figure S1B). As expected, levels of $m n b \mathrm{mRNA}$ and protein were markedly reduced by $m n b$ inhibition and by the $m n b^{G 1767}$ mutant relative to the sNPFR1-Gal4 and $w$ - controls (Figure S4B, S4C). Since sNPFR1 signaling in the insulin producing cells (IPCs) regulates body growth through insulin signaling [3], we examined the effect of $m n b$ in IPCs upon food intake. However, food intake was not affected by $m n b$ overexpression in IPGs driven via Dilp2-Gal4 (Dilp2>mnb and Dilp2>2Xmnb) or by mnb inhibition in IPCs (Dilp2>mnb-Ri) (Figure 1B). Expression of $m n b$ in sNPFR1 neurons but not in IPCs (Figure 1D-1G) is sufficient to regulate food intake.

To determine the consequences of $m n b$ control upon food intake we measured the body weight of young adults from mutant and control. Overexpression of $m n b$ in sNPFR 1 neurons $(s \mathcal{N} P F R 1>m n b)$ increased body weight relative to that of sNPFR1-Gal4 controls, similar to the effect seen when SNPFR1 is overexpressed $(s \mathcal{N} P F R 1>s \mathcal{N} P F R 1)$. On the contrary, body weight is decreased when $m n b$ is repressed in SNPFR 1 neurons $(s \mathcal{N} P F R 1>m n b-R \imath)$ and $m n b^{G 1767}$ mutant (Figure S4D). The amounts of food intake in the mutants were similar when they were normalized to body mass or to the number of flies (Figure S4E).

Since $m n b$ is involved in neural development $[6,9]$, we restricted $m n b$ expression in the adult stage using the tub-GAL80ts inducible system [18] and tested food intake. mnb overexpression (sNPFR1Gal4+tubGalo0ts $>m n b$, sNPFR1-Gal4+tubGal80ts>2Xmnb) and mnb inhibition (sNPFR1-Gal4+tubGalo0ts>mnb-Ri) flies were cultured in the $22^{\circ} \mathrm{C}$ permissive temperature until adulthood to suppress sNPFR1-Gal4 expression by the tubGalo0ts. Then, these adult flies were shifted to the $30^{\circ} \mathrm{C}$ restrictive temperature in which the tubGalo0ts cannot suppress sNPFR1-Gal4. In the permissive 
A
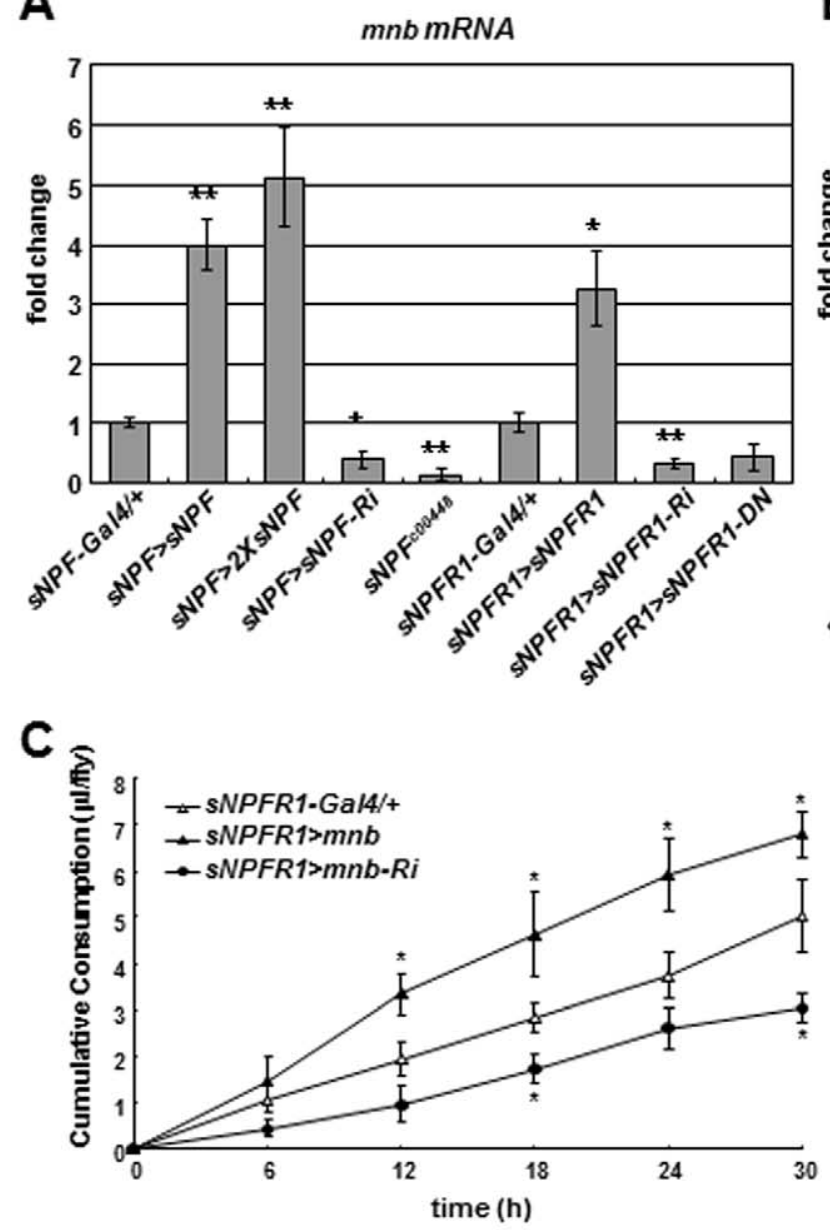
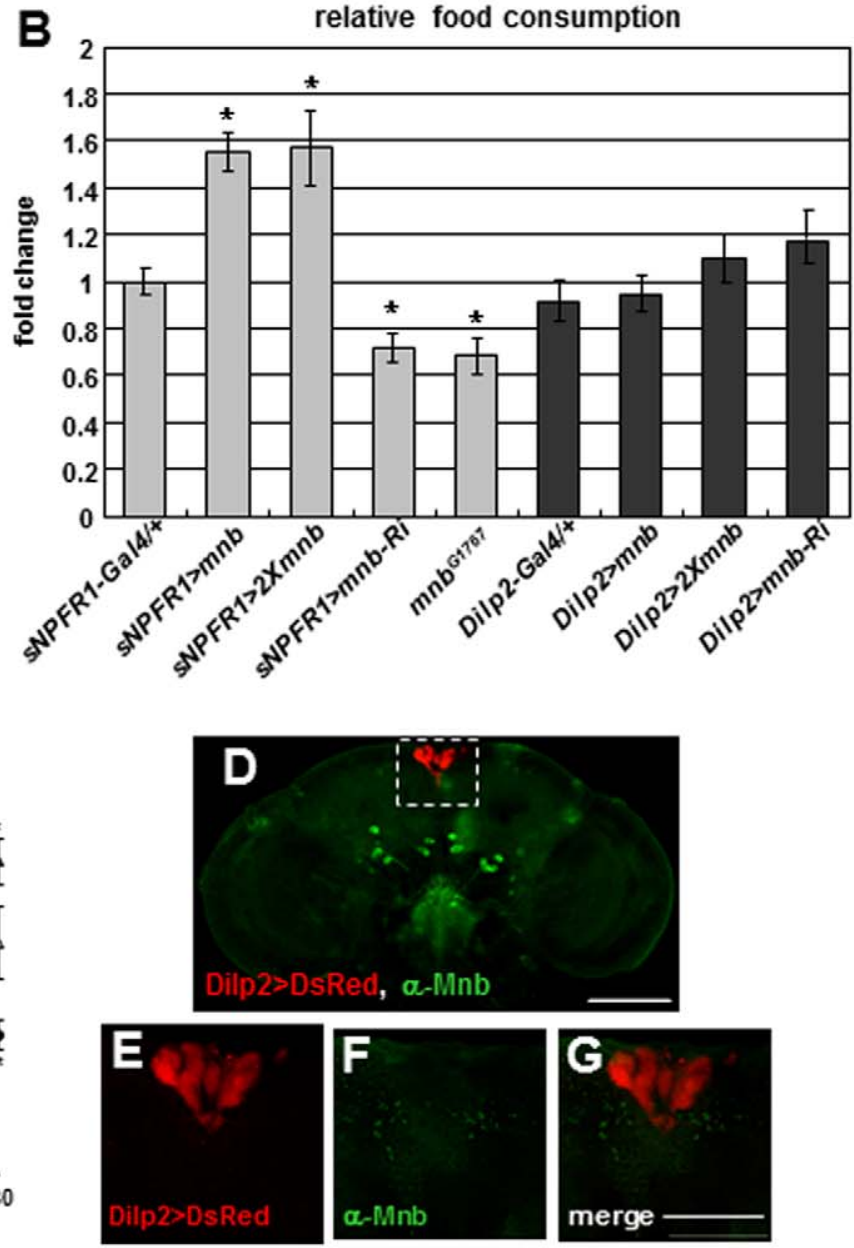
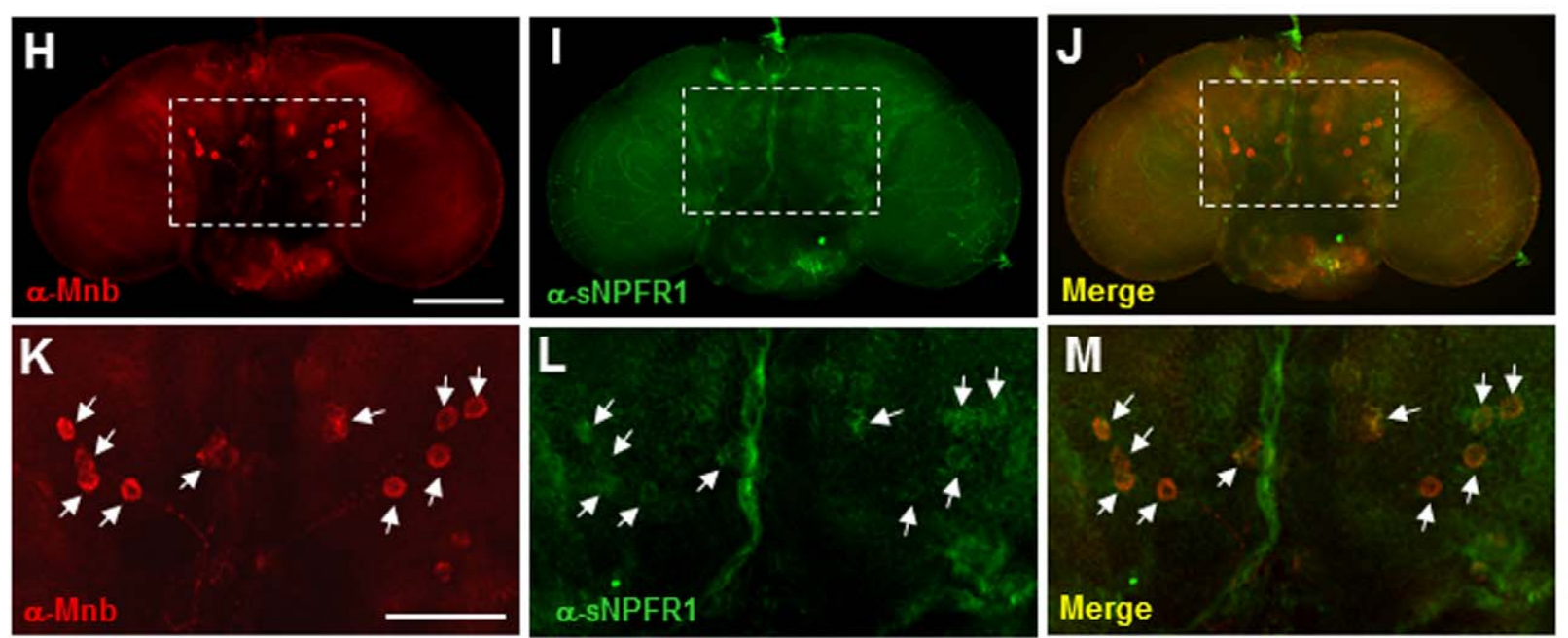

Figure 1. Expression and distribution of Drosophila mnb in adults in relation to $s N P F, s N P F R 1$, and feeding. (A) mnb mRNA prepared from fly heads was measured by RT-qPCR. mnb mRNA was increased relative to sNPF-Gal4 and sNPFR1-Gal4 controls when sNPF and sNPFR1 was overexpressed in sNPFnergic neurons ( $s N P F>s N P F$, $s N P F>2 X s N P F)$ and in sNPFR1 neurons ( $s N P F R 1>s N P F R 1)$. mnb mRNA was decreased when $s N P F$ and $s N P F R 1$ were inhibited (sNPF>sNPF-Ri, sNPF ${ }^{c 00448}$, sNPFR1>sNPFR1-Ri, sNPFR1>sNPFR1-DN). (B) Food consumption measured by the colormetric assay. Relative to sNPFR1-Gal4 control, mnb overexpression in sNPFR1 neurons (sNPFR1>mnb, sNPFR1>2Xmnb) increased feeding whereas mnb suppression (sNPFR1>mnb-Ri, mnb $b^{G 1767}$ ) decreased feeding. Overexpression or inhibition of $m n b$ in the insulin producing cells with the Dilp2-Gal4 driver (Dilp2 $>m n b$, Dilp2 $>2 X m n b$, Dilp2 $>m n b-R i)$ did not change the feeding. (C) Food consumption measured by CAFÉ assay. Relative to the sNPFR1-Gal4 (open triangle) control, sNPFR1 $>m n b$ (closed triangle) increased while $s N P F R 1>m n b-R i$ (closed circle) decreased cumulative food consumption. Data are presented as means \pm s.e.m. from three independent experiments. ${ }^{*} P<0.05,{ }^{* *} P<0.001$ (One-way ANOVA analysis). (D-G) 
Neurons of the Drosophila adult brain expressing Mnb protein (green) do not overlap with insulin producing cells marked with Dilp2 $>$ DsRed (red). (HM) Mnb protein expression neurons $(H, K$, red) and sNPFR1 protein expression neurons $(I, L$, green) were overlapped in the median neurons $(J$, dot box; $M$, arrows). Scale bars are $100 \mu \mathrm{m}(\mathrm{D}, \mathrm{H})$ and $50 \mu \mathrm{m}(\mathrm{G}, \mathrm{K})$.

doi:10.1371/journal.pgen.1002857.g001

condition, the mnb overexpression and mnb inhibition flies did not change the amount of food intake compared with the control flies (sNPFR1-Gal4; tub-Galo0ts) (Figure S5A). However, in the restrictive condition, the mnb overexpression increased food intake compared with the control and the mnb inhibition suppressed food intake (Figure S5B). These results indicate that the food intake phenotype of mnb mutants is not due to developmental effects.

\section{sNPF-PKA-CREB-mnb Signaling in Drosophila Neuronal BG2-c6 Cells}

To study how sNPFRl regulates mnb expression, we treated Drosophila central nervous system-derived BG2-c6 cells [19] with synthetic sNPF peptide, which changed $s \mathcal{N} P F$ and $s \mathcal{N P F R} 1$ expression slightly (Figure S6A). Consistent with our initial observations and with patterns in genetically manipulated flies, sNPF treatment increased $m n b$ mRNA more than 5-fold compared to the control when measured by quantitative PCR (Figure 2A). Then, we tested whether the induction of this mnb mRNA is mediated by ERK, as we have previously observed for the induction of Drosophila insulin like peptides (Dilps) by sNPF [3]. However, ERK inhibitor PD98059 treatment of the sNPF peptide-treated cells did not suppress the mnb expression. On the other hand, sNPFRl is a G-protein coupled receptor (GPCR), and the second messenger of GPCRs is cAMP or $\mathrm{Ca}^{++}$which respectively activates PKA or PKC [20]. Thus, we treated BG2c6 cells with the protein kinase A (PKA) inhibitor H89 or with protein kinase $\mathrm{G}(\mathrm{PKG})$ inhibitor Chelerythrine Chloride $(\mathrm{CG})$. H89 decreased both basal and sNPF-induced $m n b$ expression level but the PKC inhibitor CG showed no effect (Figure 2A). sNPF signaling appears to control mnb expression through PKA, not through ERK or PKC. Consistent with this interpretation, BG2-c6 cells treated with sNPF showed increased levels of cAMP in a timedependent manner, peaking at 15 min (Figure S6B).

To find the G $\alpha$ subunit of the sNPFR 1 G-protein heterotrimer,

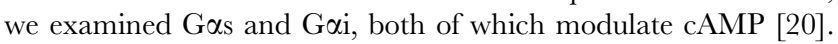
When transfected into BG2-c6 cells Gas siRNA inhibited sNPFinduced cAMP whereas transfection with G $\alpha$ i siRNA did not (Figure 2C), suggesting that $\mathrm{G} \alpha$ s is a $\mathrm{G} \alpha$ subunit of sNPFR1 that can modulate the cAMP-PKA pathway in Drosophila neuronal cells. Next, we examined the activation of the cAMP responding element binding protein (CREB), which is a PKA down-stream transcription factor [21]. sNPF stimulated the phosphorylation of CREB in control cells whereas G $\alpha$ s siRNA transfection suppressed this sNPF dependent activation of CREB (Figure 2E). In addition, G $\alpha$ s siRNA transfection completely blocked the induction of $m n b$ by sNPF, but G $\alpha$ i siRNA transfection did not (Figure 2G). These data indicate that $G \alpha$ s is a key $G \alpha$ subunit of the sNPFR 1 Gprotein as it regulates mnb expression. Taken together, these findings demonstrate that $\mathrm{NPF}$ signaling effectively regulates $m n b$ expression through the Gos-cAMP-PKA-CREB pathway in Drosophila neuronal cells.

\section{NPY-PKA-CREB-DYRK1A Signaling in Mouse Hypothalamic GT1-7 Cells}

To compare the functional conservation of sNPF-sNPFR1PKA-CREB- $m n b$ signaling with the signaling of mammalian NPY, we conducted similar experiments with mouse GT1-7 hypothalamic cells [22]. NPY treatment increased Dyrk1a mRNA while the
PKA inhibitor H89 strongly suppressed NPY-induced Dyrk1a expression (Figure 2B). NPY signaling activates Dyrk1a expression through PKA, much like the PKA mediated mnb expression by sNPF in fly neuronal cells. Next, we measured the cAMP level in the NPY treated GT1-7 cells. As expected, cAMP level increased time-dependently and peaked at 15 min (Figure S6C). Five NPY receptors (NPYR1, 2, 4, 5, and 6) mediate the NPY signal [23]. Among them, NPYR 1, 2, and 5 receptors are broadly expressed in the mouse nervous system and mediate NPY-induced food intake [24]. We treated GT1-7 cells with chemical inhibitors against these receptors: BIBO3304 for NPYR1, BIIE0246 for NPYR2, and CGP71683 for NPYR5. The NPYR1 inhibitor BIBO3304 substantially decreased the NPY-induced cAMP level; little effect was seen for the inhibitors of NPYR2 and NPYR5 (Figure 2D). Thus, NPY appears to activate the cAMP-PKA pathway mainly through NPYR 1 in GT1-7 hypothalamic cells. Next, we measured the CREB activation. As expected, inhibiting PKA or NPYR1 suppressed the NPY-induced activation of CREB (Figure 2F), confirming that NPY signal is mediated through NPYR 1-cAMPPKA-CREB. In addition, the NPYR1 inhibitor strongly suppressed NPY-induced Dyrk1a expression; this was not seen with the inhibitors of NPYR2 and NPYR5 (Figure 2H). Taken together, these findings indicate that NPY signaling regulates Dyrk1a expression mainly through the NPYR1-cAMP-PKA-CREB pathway in mouse hypothalamic cells. Importantly, this signal transduction pathway is conserved between fly neuronal cells and mammalian hypothalamic cells.

Genetic Interactions among sNPFR1, Gas, PKA, CREB, and $m n b$ Genes, and CREB ChIP Analysis

To study genetic interactions among sNPFR1, G $\alpha$, PKA, CREB, and $m n b$ genes, we suppressed $G \alpha s, P K A, C R E B$, and $m n b$ by RNAi and Dominant Negative (DN) forms in neurons that simultaneously overexpressed sNPFR1. Each of these suppression genotypes reduced the level of mnb mRNA compared with sNPFR1-Gal4 and UAS controls (Figure $3 \mathrm{~A}$ and Figure S7A). In contrast to the strong induction of $m n b$ produced by $s \mathcal{N} P F R 1$ overexpression alone (sNPFR1>sNPFR1), mnb induction was inhibited in genotypes where $s$ NPFR1 overexpression occurred with each of the suppression constructs (sNPFR1>sNPFR1+ Gas-Ri, $\quad$ sNPFR1 ${ }_{s N P F R 1+P K A-D N,} \quad s N P F R 1>s N P F R 1+C R E B-$ $D \mathcal{N}, s \mathcal{N} P F R 1>s \mathcal{N} P F R 1+m n b-R \imath$ ) (Figure $3 \mathrm{~B}$ ). In sNPFR 1 neurons of flies, as in isolated cells, Gas, PKA, and CREB may work downstream of $s$ NPFR1 to regulate $m n b$ expression. The consequences of these interactions are also seen in terms of food intake. $G \alpha s, P K A, C R E B$, and mnb suppression mutant flies have reduced food intake compared to those of the sNPFR1-Gal4 and UAS controls (Figure 3C and Figures S1C, S7B). Furthermore, increased food intake of SNPFR1 overexpression was suppressed by co-inhibition of $G \alpha s, P K A$, and $C R E B$, respectively (Figure 3D). These results suggest that the sNPFRl may regulate food intake through $G \alpha s, P K A, C R E B$, and mnb.

Based on promoter analysis of mnb genes from twelve Drosophila species, we found a conserved cAMP responding element (CRE) site (Figure S8). Interestingly, the promoters of human Dyrkla and mouse Dyrk1a genes contain CRE [25]. To test whether CREB binds to the promoter of the mnb gene, we performed the chromatin immunoprecipitation (ChIP)-PCR analysis with the CREB antibody in sNPF treated Drosophila neuronal BG2-c6 cells. 
A mnb mRNA expression in Drosophila BG2-c6

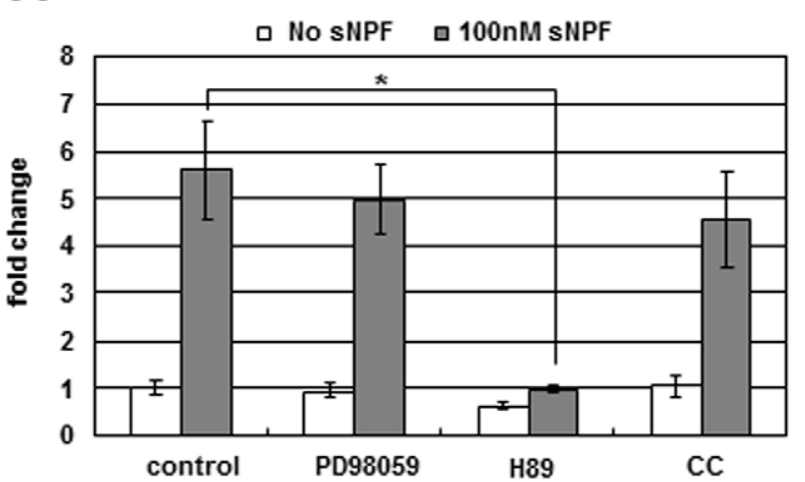

C.

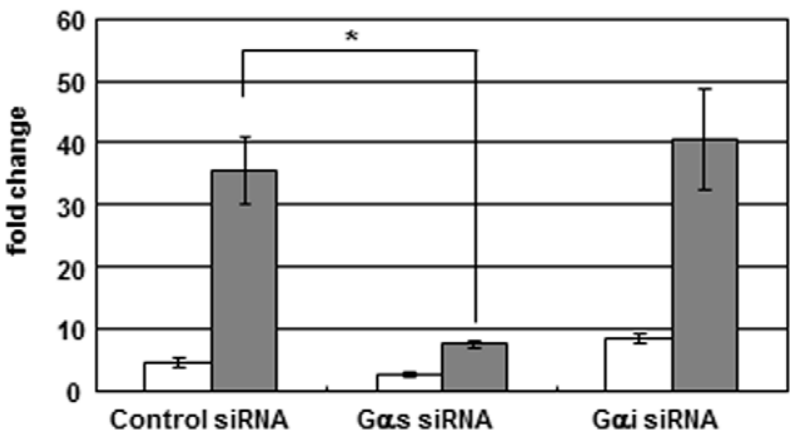

E
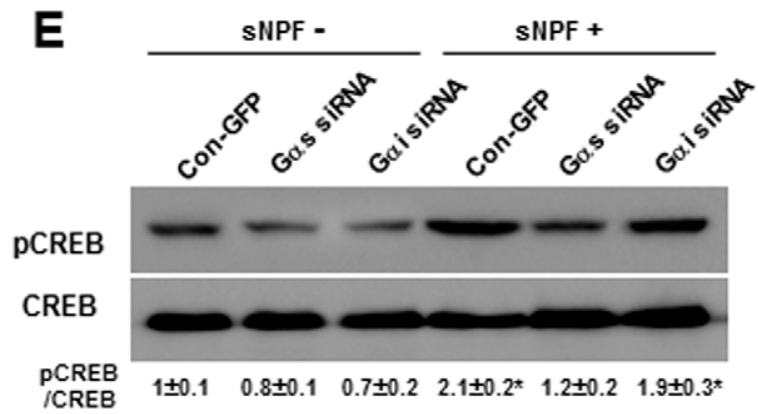

G

$m n b$ mRNA expression in BG2-c6

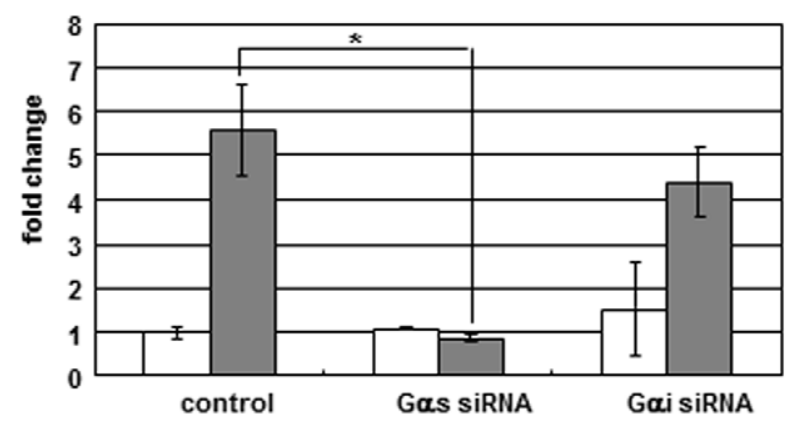

B

Dyrk1amRNA expression in mouse GT1-7

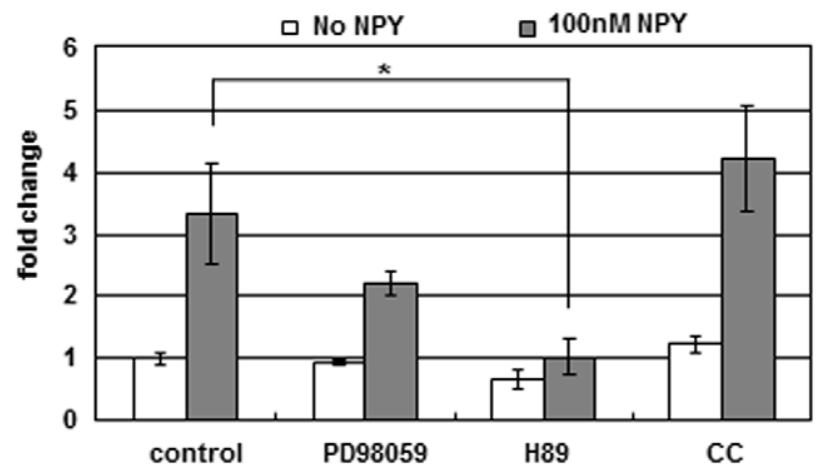

D

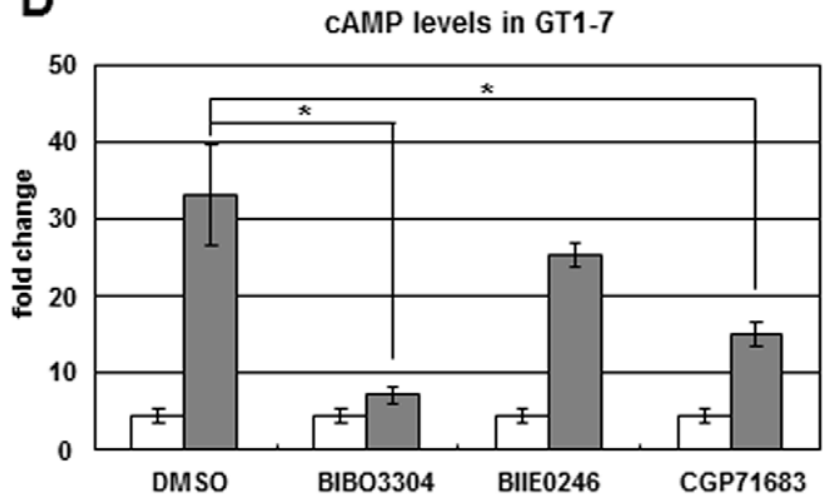

$\mathbf{F}$
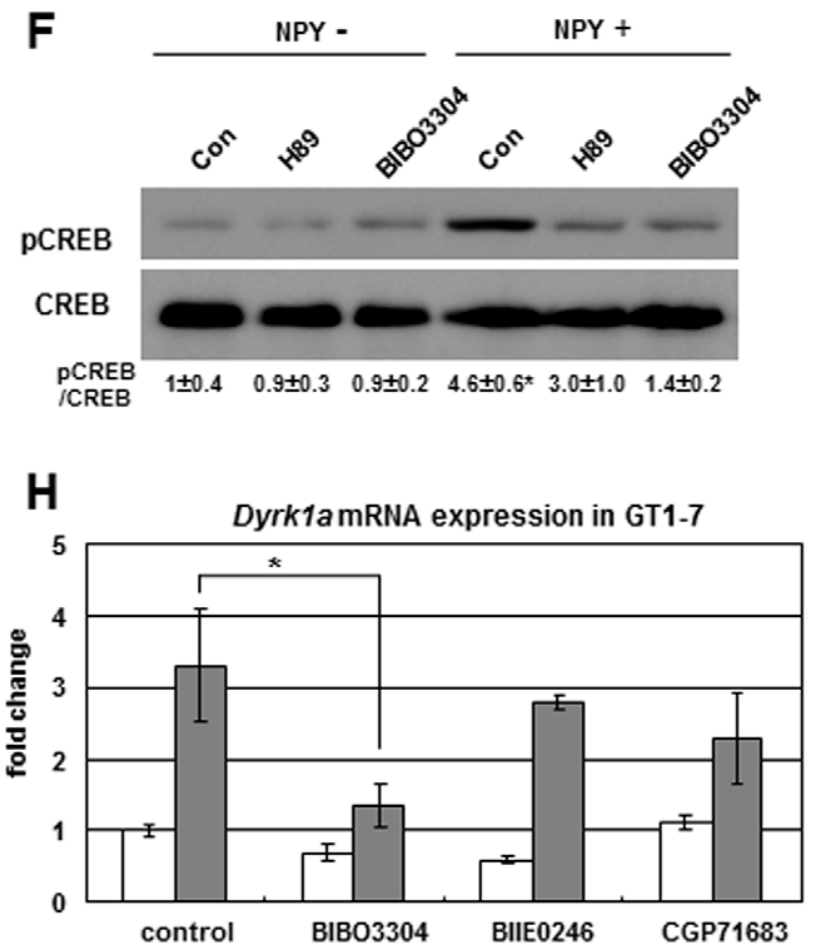

Figure 2. sNPF/NPY-sNPFR1/NPYR1-PKA-CREB-mnb/Dyrk1a signaling in Drosophila neuronal BG2-c6 cells and mouse hypothalamic GT1-7 cells. (A) mnb mRNA in Drosophila neuronal BG2-c6 cells increased in response to treatment with sNPF peptide, but not when co-treated with H89 PKA inhibitor. The ERK inhibitor PD98059 and PKC inhibitor CC did not suppress sNPF-induced mnb expression. (B) Dyrk1a mRNA in mouse hypothalamic GT1-7 cells increased in response to treatment with NPY peptide, but not when co-treated with the PKA inhibitor H89. (C) In Drosophila BG2-c6 cells, sNPF peptide induced cAMP, while transfection of cells with Gas siRNA but not Gai siRNA repressed this effect. (D) In mouse GT1-7 cells, NPY peptide induced CAMP, while co-treatment with NPYR1 inhibitor BIBO3304 but not NPYR2 and NPYR5 inhibitors strongly decreased this effect. (E) Western blot to detect activated CREB (pCREB) in Drosophila BG2-c6 cells. sNPF peptide increasd pCREB but not in cells transfected with Gas siRNA. 
(F) Western blot to detect activated CREB (pCREB) in mouse GT1-7 cells. NPY peptide increased pCREB but not when cells are co-treated with PKA inhibitor H89 or NPYR1 inhibitor BIBO3304. (G) In Drosophila BG2-c6 cells, sNPF peptide induced mnb mRNA, while transfection of cells with Gas siRNA but not G $\propto$ i siRNA repressed this effect. (H) In mouse GT1-7 cells, NPY peptide induced Dyrk1a mRNA, while co-treatment with NPYR1 inhibitor BIBO3304 but not NPYR2 and NPYR5 inhibitors strongly decreased this effect. Data are presented as means \pm s.e.m. from three independent experiments. ${ }^{*} P<0.05$ (One-way ANOVA analysis). doi:10.1371/journal.pgen.1002857.g002

CREB binding was enriched at the sNPF treated promoter region of the $m n b$ gene by 3 -fold compared to the Act5C and sNPF nontreated controls (Figure 3E). Together these in vivo and in vitro findings indicate that sNPF-sNPFR1-Gas-PKA-CREB pathway controls expression of the mnb target gene and regulates food intake in Drosophila.

\section{Positive Regulation of sNPF/NPY by the Mnb/Dyrk1a-Sir2- FOXO Pathway}

A possible avenue through which Mnb regulates food intake could involve Sirt1/Sir2. Notably, Dyrkla kinase phosphorylates Sirtl in HEK293T cells [26], and activated Sirtl deacetylates FoxOl to modulate the activity of this transcription factor in the rat hypothalamus [15]. Accordingly we determined if these interactions were present and associated in mouse hypothalamic GT1-7 cells. In cells transfected with Dyrk1a or treated with NPY, phosphorylation of Sirtl was increased as detected by immunoprecipitation with Sirtl antibody, followed by immunobloting with phospho-threonine (pThr) antibody. Sirtl phosphorylation was reduced by Dyrk1a siRNA or Dyrk1a siRNA with NPY (Figure 4A). In addition, FoxO1 acetylation was reduced in cells transfected by Dyrk1a or treated with NPY, while FoxOl acetylation was increased by Dyrk1a siRNA, Dyrk1a siRNA with NPY, or Dyrkla transfection coupled with the Sirtl inhibitor EX527 (Figure 4C). Importantly, NPY mRNA itself was increased in cells transfected with Dyrk1a or treated with NPY peptide, and NPY mRNA was decreased by Dyrkla siRNA, Dyrkla siRNA with NPY, or Dyrkla overexpression in the presence of Sirtl inhibitor (Figure 4B). In mouse hypothalamic GT1-7 cells, Dyrkla phosphorylates Sirtl and this activated Sirtl appears to deacetylate FoxOl which in turn positively regulates expression of $N P Y$.

To study genetic interactions among mnb, Sir2, and $d F O X O$ in an animal model, we manipulated Sir2 and $\triangle F O X O$ in the Drosophila $m n b$ overexpression genotype. When mnb, Sir2, and $d F O X O$ were overexpressed in sNPFR1-Gal4 neurons (sNPFR1>mnb, $s \mathcal{N P F R} 1>\operatorname{Sir} 2, s \mathcal{N} P F R 1>d F O X O)$ (Figure S9A), sNPF mRNA and food intake were increased compared to sNPFR1-Gal4 and UAS controls (Figure 4D and 4E, Figure S7B and S7C). Conversely, when $m n b$, Sir 2 , and $d F O X O$ were inhibited in $s \mathcal{N P F R} 1$ expressing neurons (sNPFR1>mnb-Ri, sNPFR1>Sir2-Ri, sNPFR1>dFOXO-Ri) (Figure S9B), the expression levels of $s \mathcal{N P F}$ and food intake were decreased or similar to those of sNPFR1-Gal4 and UAS controls (Figure 4D and 4E, Figure S7B and S7C). Finally the level of $s \mathcal{N P F}$ mRNA and food intake were reduced in adults when Sir2 or $d F O X O$ were inhibited in sNPFR 1 neurons that overexpressed $m n b$ $(s N P F R 1>m n b+S i r 2-R i$, sNPFR1>mnb+dFOXO-Ri) compared with flies only overexpressing mnb (sNPFR1>mnb). These data suggest that $m n b$ may regulate $s \mathcal{N P F}$ expression and food intake through Sir2 and $A F O X O$.

Since fasting can stimulate food intake, we tested whether an acute period of food deprivation affected the expression of $m n b$ and $s \mathcal{N} P F$ of adult flies. Levels of $m n b$ and $s \mathcal{N} P F$ mRNA increased 2-fold after $12 \mathrm{~h}$ starvation (Figure $4 \mathrm{~F}$ ). We propose that dFOXO contributes to this expression of $s \mathcal{N P F}$ in starved flies. We identified a common dFOXO consensus binding site (RWWAACA) in the sNPF promoter from twelve Drosophila species (Figure S10) and performed a chromatin immunoprecipitation (ChIP)-tiled gene array analysis with $\mathrm{dFOXO}$ antibody in fed and starved adult flies. dFOXO binding was enriched at the promoter region of $s \mathcal{N} P F$ gene more than 3-fold in the starved flies compared to the Act5c and fed controls (Figure 4G). These results suggest that the dFOXO transcriptional factor regulates $s \mathcal{N P F}$ mRNA expression by direct binding to its promoter in Drosophila, as seen for FoxOl regulation of NPY expression in mice [27].

Overall, these results from mouse hypothalamic GT1-7 cells and Drosophila indicate that the Mnb/Dyrkla-Sir2-FOXO pathway positively regulates $s \mathcal{N} P F / N P \mathcal{N}$ expression and food intake.

\section{Negative Regulation of sNPF/NPY by Insulin Signaling}

The positive feedback regulation of sNPF signaling we have described to this point must occur alongside a system to negatively regulate sNPF signaling. Insulin, one of several anorexigenic hormones, inhibits food intake through AKT-mediated FoxOl inactivation in the hypothalamus [27]. In Drosophila, neuronal overexpression of Dilps negatively regulates larval food intake [28]. To understand the inhibitory mechanism of insulin on food intake, we analyzed the phosphorylation of FOXO and the expression of $\mathcal{N P Y}$ and $s N P F$. In the mouse hypothalamic GT1-7 cells, insulin treatment increased FoxOl phosphorylation and decreased NPY mRNA while insulin combined with AKT inhibitor co-treatment slightly decreased FoxOl phosphorylation and increased NPY expression (Figure 5A, 5B). Likewise, in fly neuronal BG2-c6 cells, insulin with AKT inhibitor co-treatment increased $s \mathcal{N} P F$ mRNA (Figure 5C). Thus, in both models AKT-mediated insulin signaling increased FOXO phosphorylation and suppressed NPY or $s$ NPF expression.

We extended these results with analyses of Drosophila with insulin and insulin receptor transgenes. Compared to Dilp2-Gal4 and sNPFR1-Gal4 controls, sNPF mRNA and food intake were decreased when Dilp2 was overexpressed in insulin producing cells (Dilp2>Dilp2) and when insulin receptor (InR) was overexpressed in $s \mathcal{N} P F R 1$ expressing neurons $\left(s \mathcal{N} P F R 1>I n R^{W T}\right.$ ) (Figure 5D, 5E). On the other hand, $s \mathcal{N P F}$ expression and food intake were increased when $I n R$ was suppressed by a dominant negative construct expressed in sNPFR1 neurons (sNPFR1>InR ${ }^{D N}$ ) (Figure 5D, 5E). Fasting may contribute to $s \mathcal{N} P F$ expression and the propensity for food intake because fasting in the adult reduces the expression of several Dilps (Figure 5F), as previously observed to occur in Drosophila larvae [29].

Taken together, the results from mouse and Drosophila neuronal cells and from adult flies indicate that the insulin signaling negatively regulates $s \mathcal{N} P F / \mathcal{N} P Y$ expression and food intake.

\section{Dyrk1a TG Mice Regulate Food Intake through the FOXO- NPY Pathway}

To evaluate these Mnb/Dyrkla-Sir2-FOXO-NPY interactions and consequences in a mammalian animal model, we examined FoxOl acetylation and NPY expression in the hypothalamus of transgenic mice containing the human Dyrk1a BAC clone (hDyrk1a TG). As expected, in the Western blot, Dyrkla in the hypothalamus was increased in hDyrkla TG mice compared to controls (Figure 6A). On the other hand, FoxOl in the hypothalamus was less acetylated in hDyrkla TG mice compared to controls (Figure 6C). Hypothalamic NPY mRNA as well as serum NPY 

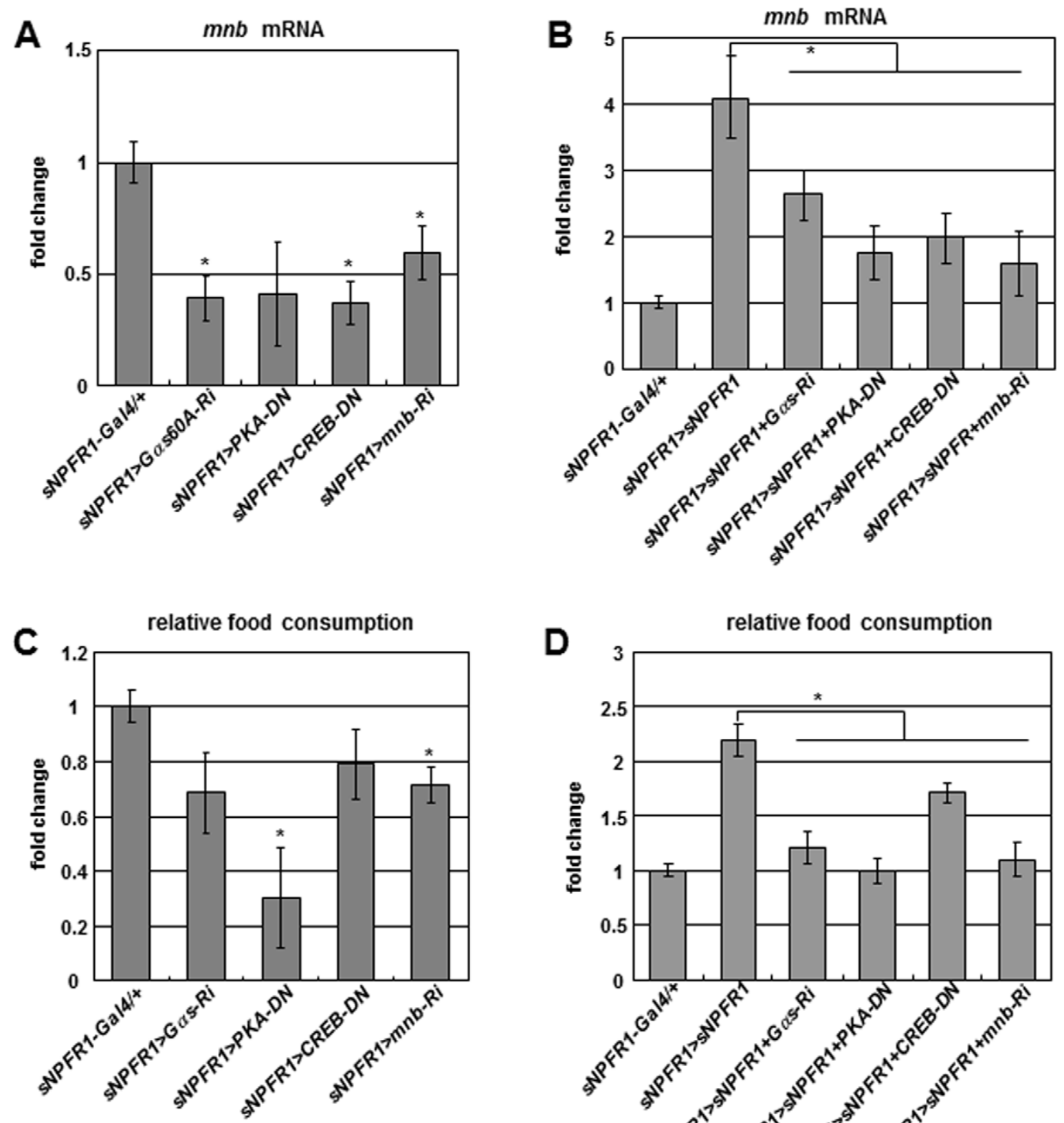

E mnb promoter enrichment in CREB ChIP
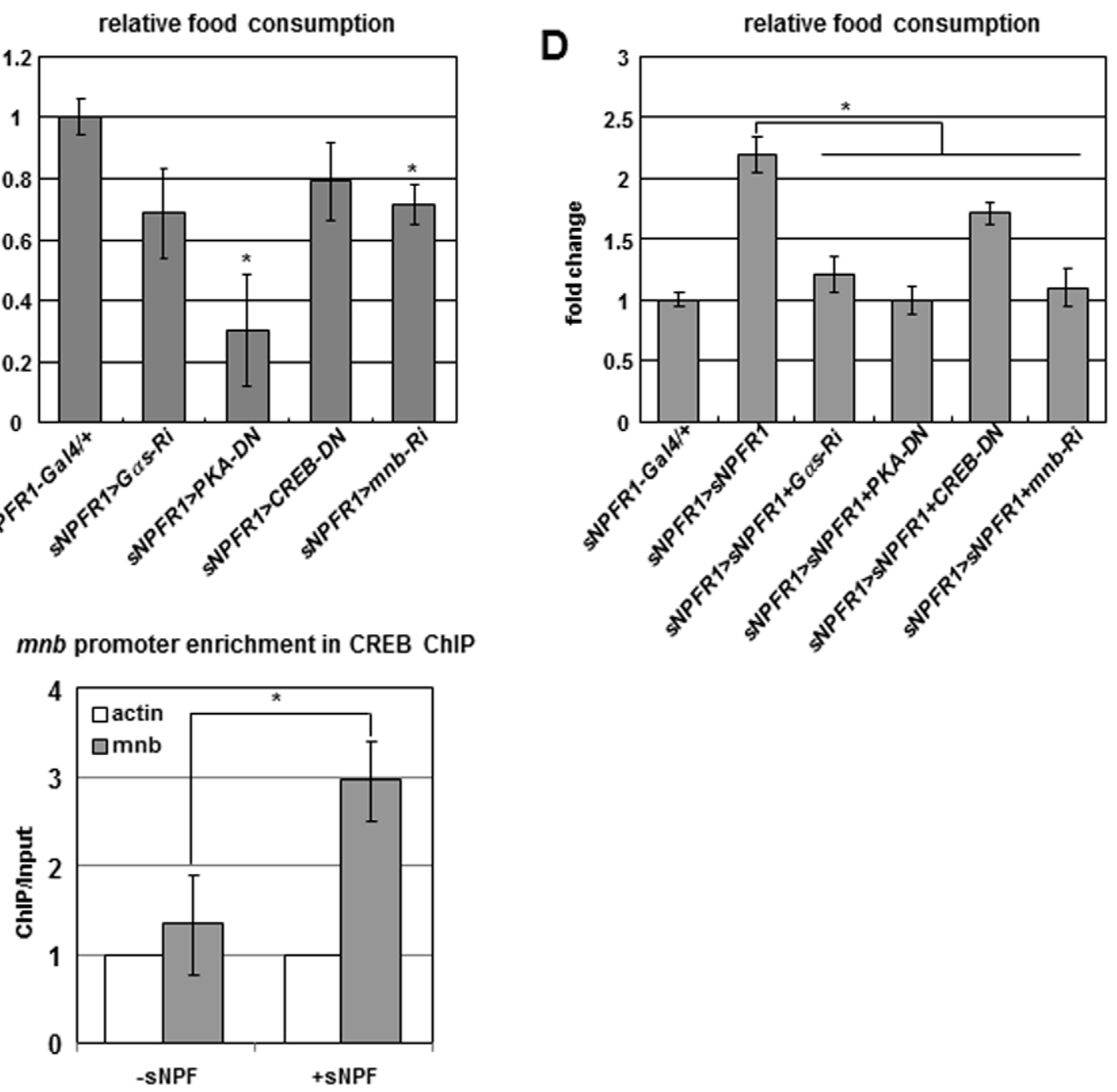
Figure 3. Genetic interactions among $s N P F R 1, G \alpha s, P K A, C R E B$, and $m n b$ genes and CREB ChIP-PCR analysis. (A, C) mnb mRNA (A) and feeding (C) were reduced by suppressing Gas, PKA, CREB, and mnb in sNPFR1 neurons relative to sNPFR1-Gal4 control. (B, D) mnb mRNA (B) and feeding (D) were reduced by suppressing Gas, PKA, CREB, and $m n b$ while overexpressing sNPFR1 in sNPFR1 neurons relative to overexpressing sNPFR1 alone (sNPFR1>sNPFR1). (E) In Drosophila BG2-c6 cells. CREB binding was enriched at the promoter region of the mnb gene by 3-fold compared to the $A c t 5 c$ and sNPF peptide non-treated controls (ChIP-PCR). Data are presented as means \pm s.e.m. from three independent experiments. ${ }^{*} P<0.05$ (Oneway ANOVA analysis).

doi:10.1371/journal.pgen.1002857.g003

levels were elevated in in hDyrk1a TG mice compared to controls (Figure 6B). Thus, mammalian Dyrk la appears to regulate FoxO1 acetylation and NPY expression in the mouse hypothalamus, as we have observed for this system in Drosophila sNPFR1 neurons.

To assess whether mammalian Dyrkla also regulates food intake as seen for the homolog mnb of Drosophila, we monitored food intake in seven-week-old hDyrk1a TG mice. Daily food consumption was increased in the transgenic mice compared to littermate controls (Figure 6D) and the average food intake of hDyrkla transgenic mice was elevated by $15 \%$ (Figure $6 \mathrm{E}$ ). Correspondingly, the hDyrkla transgenic mice presented slightly increased mass (Figure S11). Dyrk1a thus appears to regulate food intake through the expression of NPY mediated by FOXO in a molecular pathway that is evolutionarily conserved in Drosophila.

\section{Discussion}

The production of $\mathrm{SNPF}$ and NPY in sNPFnergic and hypothalamic neurons of flies and mammals respectively, is increased during fasting. These neuropeptides are secreted to produce paracrine and endocrine effects [24] but also feedback upon their synthesizing neurons where they respectively induce mnb and Dyrk1a gene expression through the PKA-CREB pathway (Figure 6F). This Mnb/Dyrkla kinase phosphorylates and activates the Sir2/Sirtl deacetylase, which in turn deacetylates and activates the FOXO transcription factor. Among its many potential targets, FOXO then increases $s N P F / N P Y$ mRNA expression. Negative controls modulate the positive feedback of sNPF/NPY. Feeding activates the insulin receptor-PI3K-AKT pathway. FOXO becomes phosphorylated and transcriptionally inactivated by translocation to the cytoplasm [30]. In this state the induction of $s \mathcal{N} P F / \mathcal{N P Y}$ by FOXO is decreased. Because sNPF and NPY are orexogenic, their positive feedback during fasting should reinforce the propensity for food intake whereas the negative regulation of $s \mathcal{N P F}$ and $\mathcal{N P Y}$ mRNA during feeding condition would then contribute to satiety (Figure 6F).

FOXO family transcriptional factors are involved in metabolism, longevity, and cell proliferation [31]. FOXO is in part regulated in these processes by post-transcriptional modifications including phosphorylation and acetylation [30]. In many model systems, the ligand activated Insulin-PI3K-AKT pathway phosphorylates FOXO to inactivate this transcription factor by moving it to the cytoplasm. The cytoplasmic localization of FOXO is mediated by 14-3-3 chaperone proteins in Drosophila and mammals $[32,33]$. FOXO may also be acetylated, as is FoxOl of mice, by the CREB-binding protein (CBP)/p300 acetylase and this inhibits FOXO transcriptional function by suppressing its DNA-binding affinity. Such FoxOl acetylation can be reversed by SirT1 to help activate the FoxOl transcription factor [34]. Here we describe for Drosophila how dFOXO in sNPFRl neurons regulates the expression of $s \mathcal{N P F}$ and food intake (Figure 4D, 4E). This mechanism parallels how hypothalamic FoxOl regulates food intake through its control of orexigenic NPY and Agrp in rodents $[12,27]$. Post-transcriptional modification of FOXO is central to these controls in both animals. $s \mathcal{N} P F$ and $\mathcal{N P Y}$ expression is increased when FOXO is deacetylated by Sir2/Sirt1, while $s \mathcal{N P F}$ and NPY are decreased when FOXO is phosphorylated via the
Insulin-PI3K-AKT pathway. Post-transcriptional modifications of FOXO proteins play a critical role for controlling food intake through the $s \mathcal{N} P F$ and $\mathcal{N P Y}$ expression in flies and rodents.

Mnb/Dyrkla has been described to participate in olfactory learning, circadian rhythm, and the development of the nervous system and brain [6]. Mnb and Dyrkla proteins contain a nuclear targeting signal sequence, a protein kinase domain, a PEST domain, and a serine/threonine rich domain. The kinase domains are evolutionary well-conserved from flies to humans [35]. In Down syndrome (DS), chromosome 21 trisomy gives patients three copies of a critical region that includes the Mnb/Dyrkla; trisomy of this region is associated with anomalies of both the nervous and endocrine systems [36]. DS patients often show high Body Mass Index due to the increased fat mass. Children with DS have elevated serum leptin coupled with leptin resistance, both of which contribute to the obesity risk common to DS patients $[37,38]$. We now observe a novel function of Mnb/Dyrkla that may underlay this metabolic condition of DS patients. Mnb/Dyrkla regulates food intake in flies and mice. This is controlled by sNPF/NPYPKA-CREB up-stream signaling and thus produces down-stream affects upon Sir2/Sirt1-FOXO-sNPF/NPY. Fasting not only increases the expression of $m n b$, but also of $s \mathcal{N P F}$, suggesting that Mnb kinase activates a positive feedback loop where Sir2-dFOXO induces $s N P F$ gene expression. Notably, fasting increases Sirtl deacetylase activity and localizes FoxOl to the nucleus in the orexogenic AgRP neurons of the mouse hypothalamus [15]. Increased dosage of Dyrk1a in DS patients may reinforce the positive feedback by NPY and disrupt the balance between hunger and satiety required to maintain a healthy body mass.

Insulin produced in the pancreas affects the hypothalamus to regulate feeding in mammals [1]. Insulin injected into the intracerebroventrical of the hypothalamus reduces food intake while inhibiting insulin receptors of the hypothalamic ARC nucleus causes hyperphasia and obesity in rodent models $[39,40]$. Here we saw a similar pattern for Drosophila where overexpression of insulin-like peptide (Dilp2) at insulin producing neurons decreased food intake while food intake was increased by inhibiting the insulin receptor in sNPFR 1 expressing neurons (Figure 5E). Likewise, during fasting, serum insulin and leptin levels are decreased in mammals [1], as is mRNA for insulin-like peptides of Drosophila [29,41] (Figure 5F). Thus, the mechanism by which insulin and insulin receptor signaling suppresses food intake is conserved from fly to mammals in at least some important ways.

Previously, we reported how sNPF signaling regulates Dilp expression through ERK in IPCs and controls growth in Drosophila [3]. Here, we show that sNPF signaling regulates $m n b$ expression through the PKA-CREB pathway in non-IPG neurons and controls food intake (Figure 1B, 1D-1G). Since sNPF works through the sNPFRl receptor, sNPFRl in IPCs and non-IPCs neurons might transduce different signals and thereby modulate different phenotypes. Four Dilps (Dilp1, 2, 3, and 5) are expressed in the IPCs of the brain [42]. Interestingly, levels of Dilp1 and 2 mRNA are reduced in the $s N P F$ mutant, which has small body size [3], but here we find only Dilp3 and 5 mRNA levels are reduced upon $24 \mathrm{~h}$ fasting. Likewise, only Dilp 5 is reduced when adult flies are maintained on yeast-limited diets [43]. In addition, Dilp1 and 2 


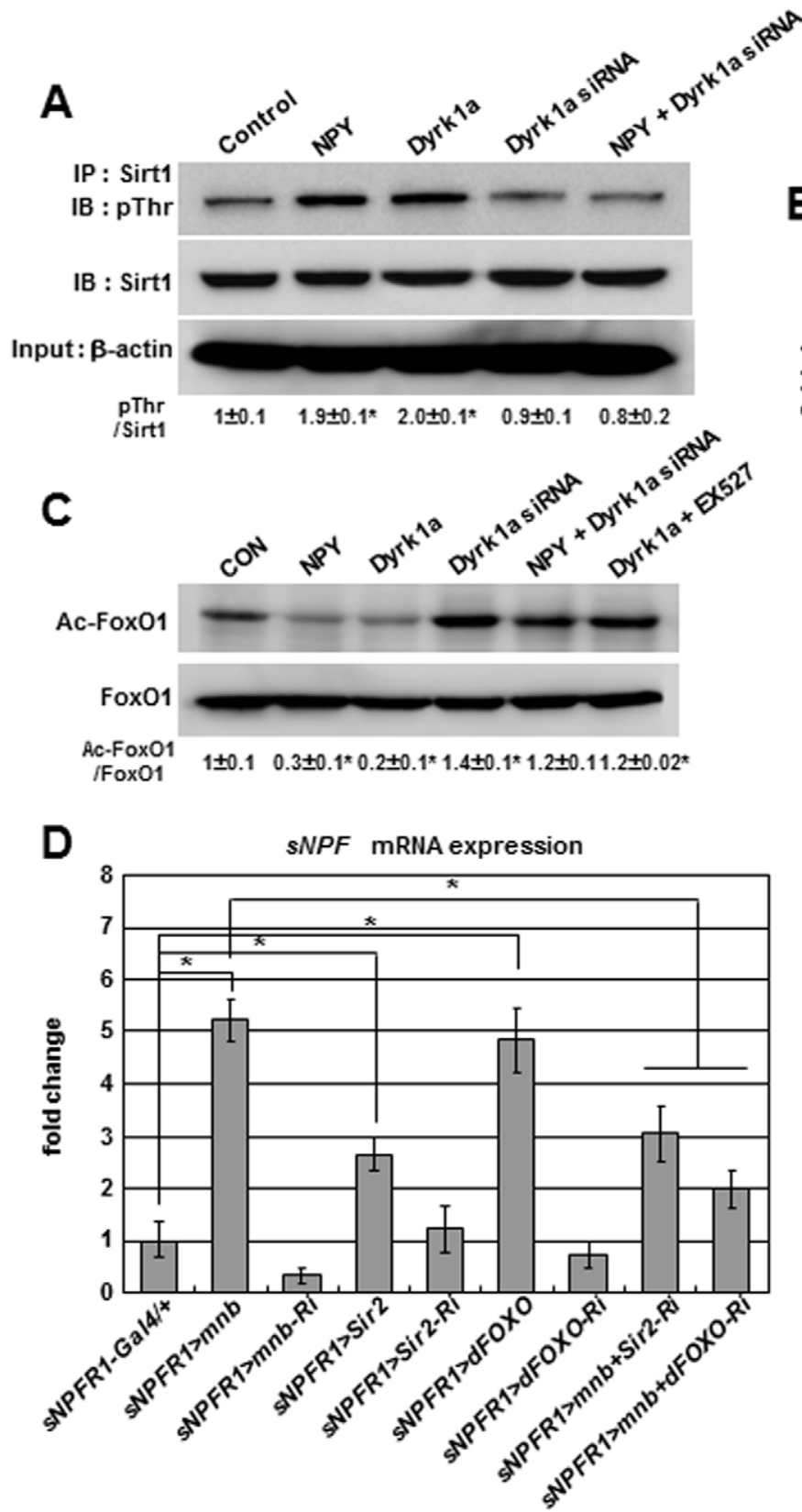

$\mathbf{F}$

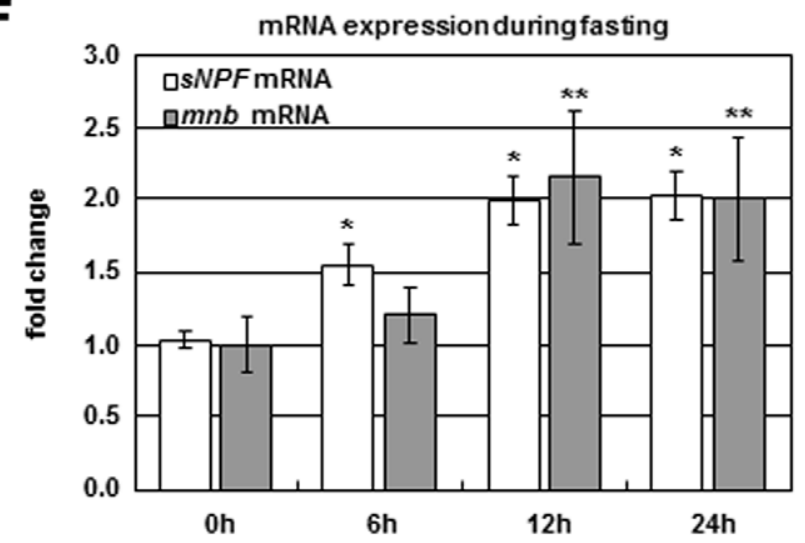

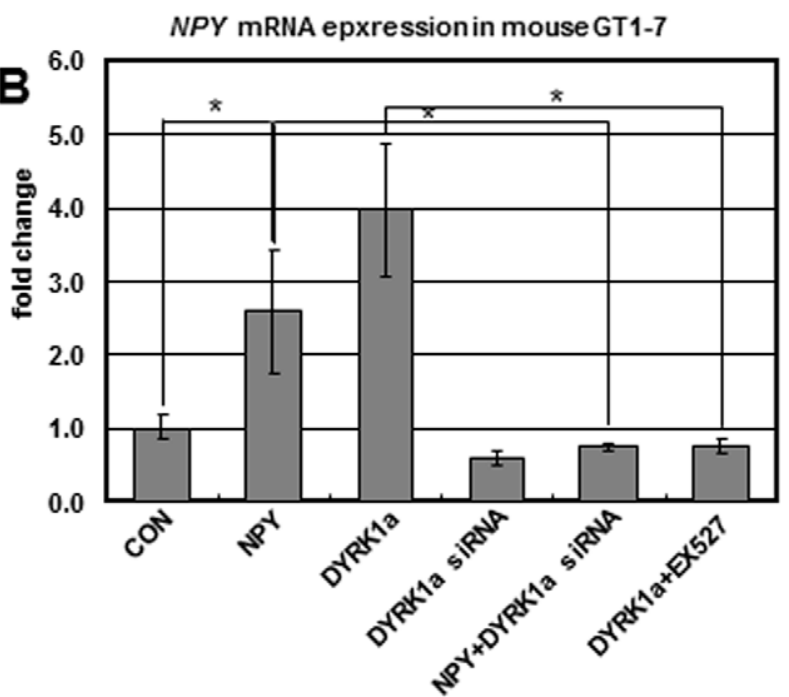

E

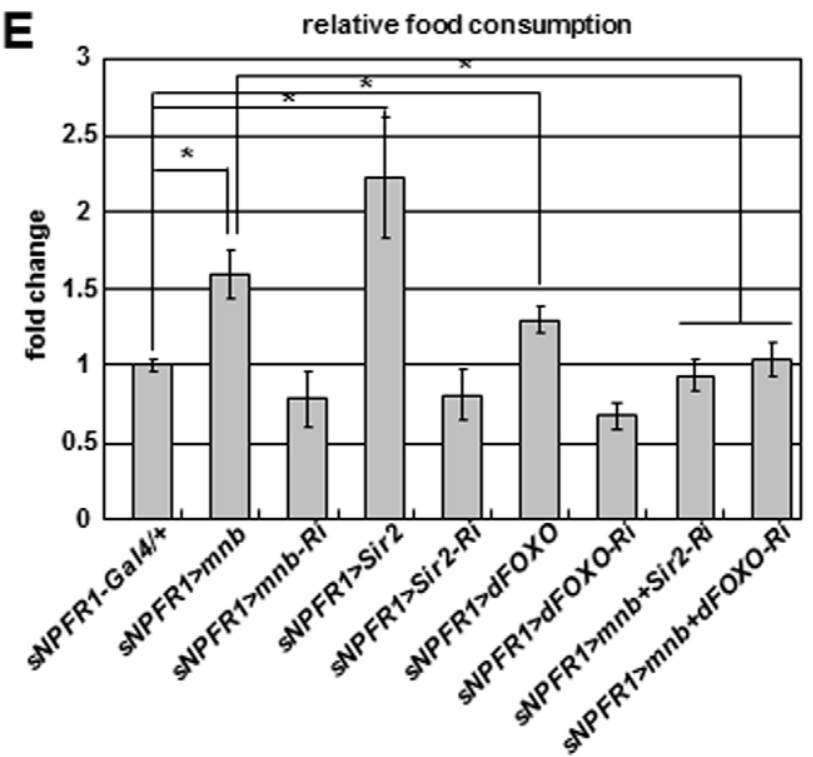

G sNPF promoter enrichment in dFOXO ChIP

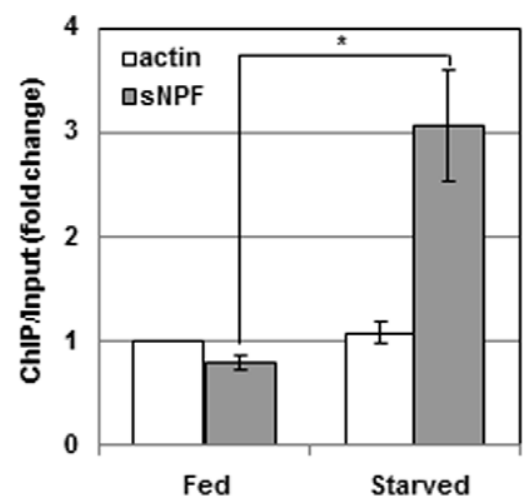


Figure 4. Positive regulation of $s N P F / N P Y$ by the Mnb/Dyrk1a-Sir2-FOXO pathway. (A) Sirt1 phosphorylation was increased in mouse GT1-7 cells transfected with Dyrk1a or treated with NPY but reduced in cells transfected with Dyrk1a siRNA or Dyrk1a siRNA co-treated with NPY. (B) NPY mRNA was increased in GT1-7 cells transfected with Dyrk1a or treated with NPY peptide, but reduced in cells transfected with Dyrk1a siRNA, Dyrk1a siRNA co-treated with NPY, or Dyrk1a co-treated with Sirt1 inhibitor EX527. (C) FoxO1 acetylation was reduced in GT1-7 cells transfected with Dyrk1a or treated with NPY peptide, but FoxO1 acetylation was increased in cells transfected with Dyrk1a siRNA, Dyrk1a siRNA co-treated with NPY, or Dyrk1a co-treated with Sirt1 inhibitor EX527. (D, E) sNPF mRNA (D) and food intake (E) were reduced when Sir2 or dFOXO were inhibited while mnb was overexpressed in $s N P F R 1$ expressing neurons relative to levels observed for $m n b$ overexpression alone (sNPFR1>mnb). (F) $s N P F$ and $m n b$ mRNA increased in adults starved $12 \mathrm{~h}$. (G) dFOXO binding to the promoter region of the $S N P F$ gene in adult flies starved $12 \mathrm{~h}$ was elevated relative to the $A c t 5 c$ and fed controls (ChIP-chip). Data are presented as means \pm s.e.m. from three independent experiments. ${ }^{*} P<0.05$, ${ }^{* * P}<0.001$ (One-way ANOVA analysis).

doi:10.1371/journal.pgen.1002857.g004

null mutants show slight reduced body weights but Dilp3 and Dilp5 null mutants do not [44]. These results suggest that Dilp1 and 2 behave like a mammalian insulin growth factor for size regulation while Dilp 3 and 5 act like a mammalian insulin for the regulation of metabolism. However, in the long term starvation, Dilp2 and Dilp 5 mRNA levels are reduced and Dilp3 mRNA expression is increased [45].

During fasting, sNPF but not sNPFR1 mRNA expression was increased in samples prepared from fly heads (Figure $4 \mathrm{~F}$ and Figure S9C), which increases food intake. On the other hand, in feeding, the high level of insulin signaling reduced $s \mathcal{N} P F$ but not sNPFR1 mRNA expression and suppressed food intake (Figure 5D and 5E, Figure S9D). Interestingly, in the antenna of starved flies, sNPFR1 but not $s N P F$ mRNA expression is increased and induces presynaptic facilitation, which resulted in effective odor-driven food search. However, high insulin signaling suppresses sNPFR1 mRNA expression and prevents presynaptic facilitation in DMl glomerulus [46]. These results indicate that starvation-mediated or insulin signaling-mediated sNPF-sNPFRl signaling plays a critical role in Drosophila feeding behavior including food intake and food search even though the fine tuning is different.

In this study, we present a molecular mechanism for how sNPF and NPY regulate food intake in Drosophila and mice. We describe a system of positive feedback regulation for sNPF and NPY signaling that increases food intake and a mode of negative regulation for $\mathrm{SNPF}$ and NPY by the insulin signaling that suppresses food intake. Modifications of the FOXO protein play a critical role for regulating $s \mathcal{N} P F$ and $\mathcal{N P Y}$ expression, resulting in the control of food intake.

\section{Materials and Methods}

\section{Drosophila Culture and Stocks}

Drosophila melanogaster were cultured and at $25^{\circ} \mathrm{C}$ on standard cornmeal, yeast, sugar, agar diet. Wild-type Canton-S, w', and UAS$C R E B-D \mathcal{N}$ were obtained from the Bloomington stock center. $s \mathcal{N P F}{ }^{00448}$ was obtained from the Harvard stock center (Exelixis stock collection). UAS-sNPF, UAS-2XsNPF, UAS-sNPF-Ri, UASsNPFR1, UAS-sNPFR1-DN and sNPF-Gal4 transgenic flies were described in our previous reports $[2,3,16]$. The sNPFR1-Gal4 construct was generated from a $2.5 \mathrm{~kb}$ genomic DNA fragment of the $5^{\prime}$-untranslated region of the sNPFR1 gene. The full-length coding sequence of Drosophila minibrain-H (mnb, CG 42273) was subcloned into the $p U A S$ vector to generate the $p U A S-m n b$ construct. sNPFR1-Gal4 and UAS-mnb transgenic flies were obtained by the P-element-mediated germ line transformation [47]. $m n b^{G 1767}$, an EP line for minibrain, was purchased from the GenExel, Inc. (KAIST, Korea). UAS-sNPFR1-Ri (VDRC9379), UAS-mnb-Ri (VDRC28628), UAS-Sir2-Ri (VDRC23201) and UASFOXO-Ri (VDRC106097) were obtained from the Vienna Drosophila RNAi Center (VDRC). Dilp2-Gal4, UAS-Gas-Ri, UAS$P K A-D \mathcal{N}$ (a dominant-negative form of PKA), UAS-Sir2 transgenic flies were described previously $[42,48,49,50,51]$. To express these
UAS lines, UAS-Gal4 system was used [52]. For minimizing the genetic background effect among tested Drosophila lines, all stocks were crossed with $w$ - and then crossed to the second $(w-; B c, E l p)$ $C y O)$ or third $(w-; D / T M 3, S b)$ chromosome balancers, respectively. For making double mutants, $w-; T(2: 3) A p^{X a} / C y O$; TM3 was crossed with the flies containing $U A S-X$ transgene to produce $w$-; $U A S-X / C y O ;+/ T M 3$. Then, w-; +/CyO; UAS-Y/TM3 flies generated by the similar way were crossed with w-; $U A S-X / C y O$; $+/ T M 3$ to produce $w_{-}$; UAS-X/CyO; UAS-Y/TM3.

\section{Cell Culture, Stimulation, and Transfection}

Drosophila BG2-c6 cells established by the single colony isolation of primary cells derived from the third instar larval central nervous system. This cell line synthesizes acetylcholine and expresses insect neuron specific glycans and a RNA-binding protein Elav [19]. BG2-c6 cells purchased from the Drosophila Genomics Resource Center (DGRC, Indiana University) were maintained at $26^{\circ} \mathrm{C}$ in Schneider medium supplemented with $10 \%$ bovine calf serum. Immortalized GT1-7 mouse hypothalamic neurons [22] were cultured in $4.5 \mathrm{~g} / \mathrm{l}$ glucose Dulbecco's modified Eagle's medium (DMEM) supplemented with 10\% fetal bovine serum, $2 \%$ of $1-$ glutamine, $100 \mu \mathrm{U} / \mathrm{ml}$ penicillin and $100 \mu \mathrm{g} / \mathrm{ml}$ streptomycin in $5 \% \mathrm{CO}_{2}$ at $37^{\circ} \mathrm{C}$. The culture medium was changed every $2-3$ days. Before peptide treatments, cells were starved for $8 \mathrm{~h}$ in the serum-free medium containing $0.5 \% \mathrm{BSA}$ and pretreated with a chemical inhibitor or vehicle. PKA inhibitor H89 $(10 \mu \mathrm{M}$, Calbiochem), ERK-specific kinase MEK inhibitor PD98059 (10 $\mu \mathrm{M}$, Calbiochem), PKG inhibitor Chelerythrine chloride (1 $\mu \mathrm{M}$, Sigma) were used. NPY1R inhibitor BIBO3304 (10 nM), NPY2R inhibitor BIIE0246 (50 nM), NPY5R inhibitor CGP71683 $(1 \mu \mathrm{M})$ and Sirtl inhibitor EX527 $(10 \mu \mathrm{M})$ were purchased from Tocris. Then, cells were treated with $100 \mathrm{nM}$ synthetic 19 amino acids sNPF2 or $100 \mathrm{nM}$ human NPY 1-36 peptide (Sigma). For transfection, cells were cultured in the growth medium without antibiotics and transfected with small interfering RNA (siRNA) using Lipofectamine 2000 reagent (Invitrogen). Gas and $G \alpha i$ siRNA constructs were designed by the BLOCK-iT RNAi Designer and Dyrk1a siRNA was purchased from Invitrogen. The sequences of siRNA are caggauauucuucggugccguguuu for $\mathrm{G} \alpha \mathrm{s}$

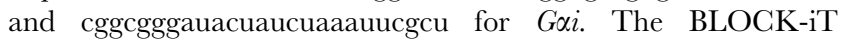
Fluorescent Oligo, which is a fluorescent-labeled dsRNA oligomer, was used as the non-targeting siRNA control. For the overexpression mouse Dyrkla, a full-length mDyrkla cDNA was cloned to pCDNA3.1 (Invitrogen).

\section{Drosophila Food Intake Assay}

We measured food intake of Drosophila in two ways. The CAFE assay [17] was performed with 3 day-old adult male flies. Twelve hours before the assay, ten flies were placed in the CAFE device [17] containing 5\% sucrose solution in calibrated glass micropipettes (VWR, West Chester, PA). At time zero, the micropipettes with $5 \%$ sucrose solution were replaced and the amount of liquid consumed was measured every $6 \mathrm{~h}$. A colorimetric food intake 
A

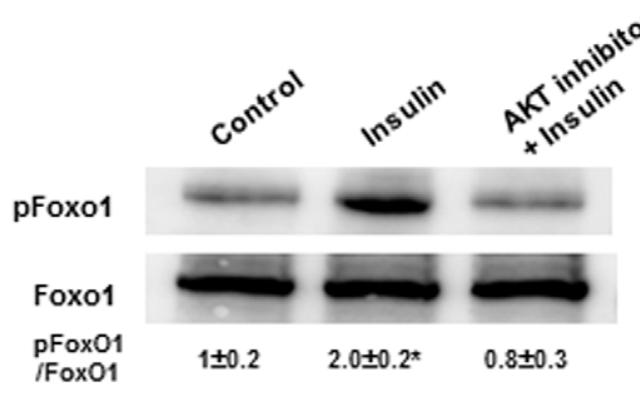

C

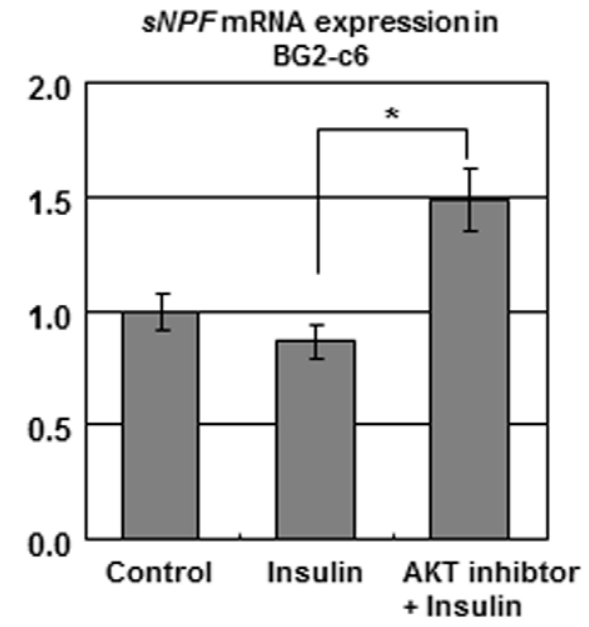

E

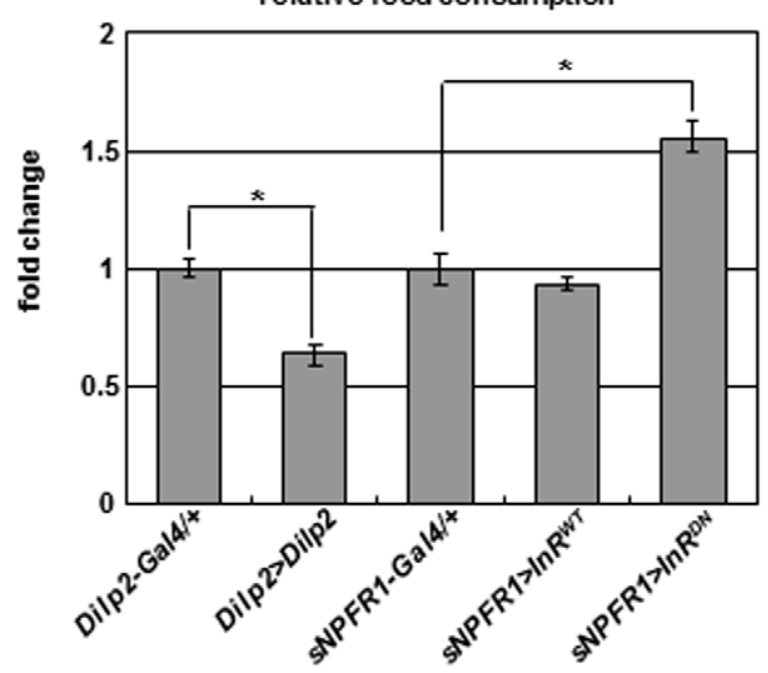

B

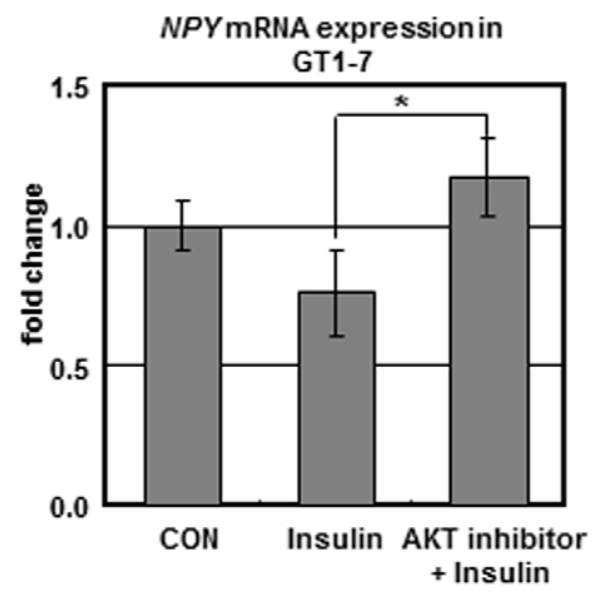

D

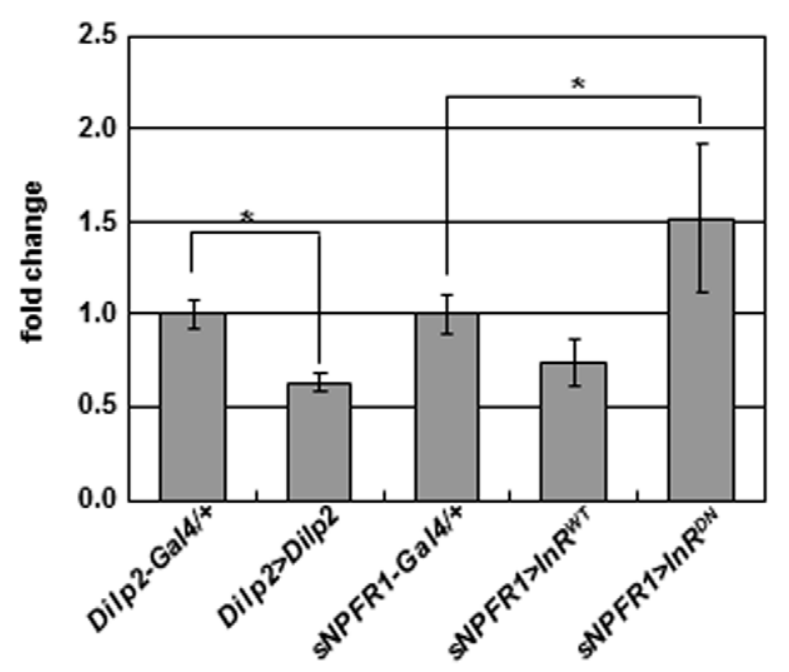

F

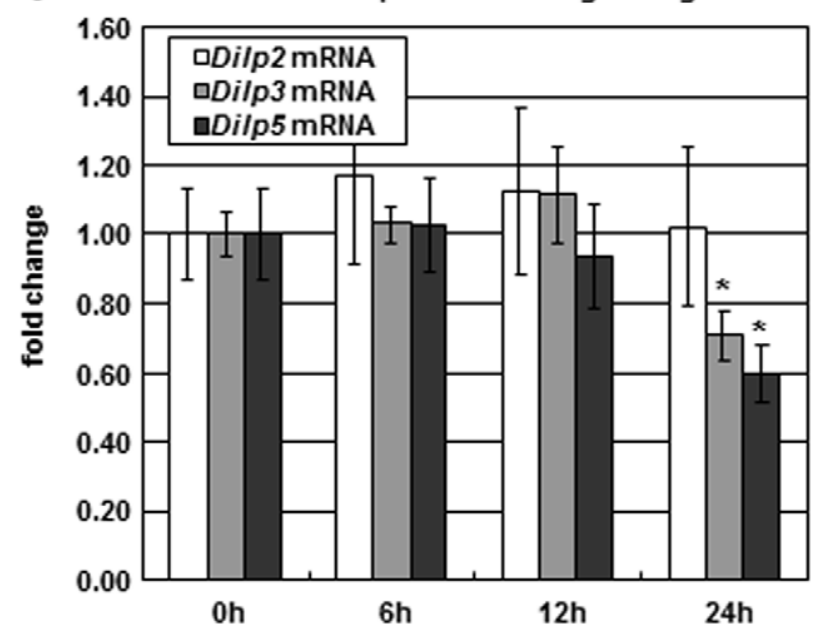

Figure 5. Negative regulation of $s N P F / N P Y$ by insulin signaling. (A, B) Insulin treatment increased FoxO1 phosphorylation and decreased $N P Y$ expression in mouse hypothalamic GT1-7 cells while insulin with AKT inhibitor co-treatment slightly decreased FoxO1 phosphorylation and increased $N P Y$ expression. (C) Insulin with AKT inhibitor co-treatment increased sNPF expression in fly neuronal BG2-c6 cells. (D, E) sNPF expression (D) and food intake (E) were decreased in adult flies overexpressing Dilp2 in IPCs (Dilp2>Dilp2) and overexpressing insulin receptor (InR) in sNPFR1 neurons $\left(s N P F R 1>\operatorname{In} R^{W T}\right)$, while $s N P F$ mRNA and food intake increased when $\ln R$ was suppressed in $s N P F R 1$ neurons $\left(s N P F R 1>\ln R^{D N}\right)$. (F) Fasting $($ at $24 \mathrm{~h})$ reduces Dilp3, and Dilp 5 mRNA but not Dilp2 mRNA. Data are presented as means \pm s.e.m. from three independent experiments. ${ }^{*} P<0.05$ (One-way ANOVA analysis).

doi:10.1371/journal.pgen.1002857.g005 

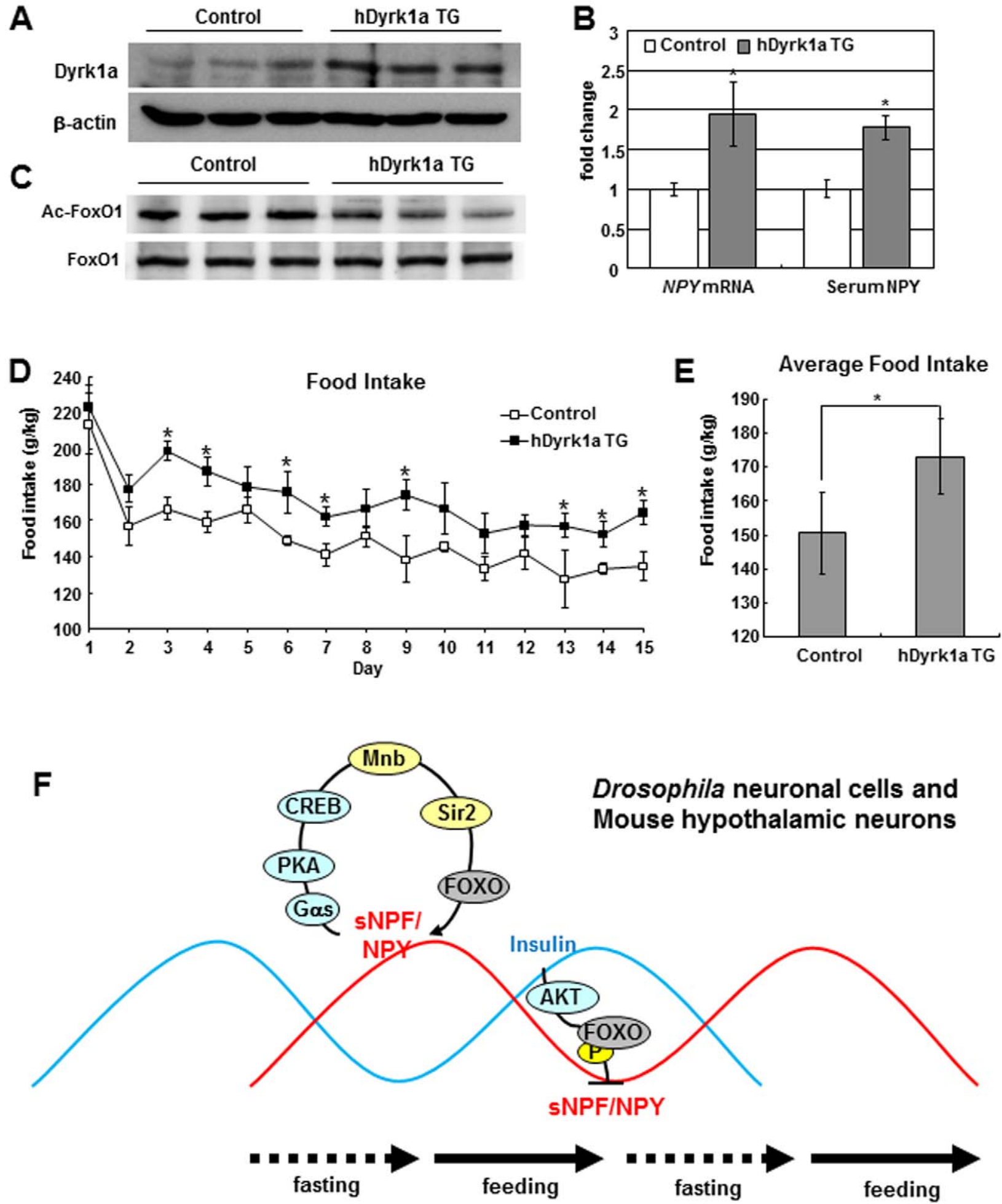

Figure 6. hDyrk1a transgenic mice regulate food intake through the FOXO-NPY pathway. (A, C) In the hypothalamus of $h D y r k 1 a$ transgenic mice, Dyrk1a was increased and FoxO1 acetylation was reduced compared with those of the littermate control mice. (B) NPY mRNA from hypothalamus and serum NPY were increased in $h D y r k 1 a$ transgenic mice. (D) Daily food intake was increased in $h D y r k 1 a$ transgenic mice compared with the littermate controls. (E) Average food intake of $h$ Dyrk1a transgenic mice increased by $15 \%$. Data are presented as means \pm s.e.m. from three independent experiments. ${ }^{*} P<0.05$ (One-way ANOVA analysis). (F) The model of this study. doi:10.1371/journal.pgen.1002857.g006 
assay was modified from published methods [2,53]. Since flies had most fed color food in the crop during first $30 \mathrm{~min}$ and started to excrete from $1 \mathrm{~h}$ (Figure S4C, S4D) [54], flies were starved in PBS-containing vials for $2 \mathrm{~h}$ and fed for $30 \mathrm{~min}$ in vials containing $0.05 \%$ Bromophenol Blue dye and 10\% sucrose in yeast paste. Then, the flies were frozen, homogenized in PBS, and centrifuged twice for $25 \mathrm{~min}$ each. The supernatant was measured at $625 \mathrm{~nm}$. Each experiment consisted of 20 flies, and the assay was repeated at least three times.

\section{Mouse Food Intake Assay}

Dyrkla transgenic mice expressed the human Dyrk1a BAC clone in the $C 57 B L / 6$ background [55]. Seven weeks-old male Dyrk1a TG and littermate control mice were used in the experiments $(n=7)$. The mice were housed individually in the standard plastic rodent cages. They were maintained at $22 \pm 2^{\circ} \mathrm{C}$ in a room with a 12-hour light/dark cycle and habituated to frequent handling. Food intake and body weight were measured within 30 min before the light turned on and off. Drinking water was available at all times. Food intake data were corrected with body weight.

Animal care and all experiments were conducted according to KRIBB Guidelines for the Care and Use of Laboratory Animals and Inje University Council.

\section{Drosophila Starvation}

Twenty $w$-female flies were starved overnight and fed for $2 \mathrm{~h}$ for the physiological synchronization. Then, starvations for the experiment were started. The heads from starved flies were collected for the Quantitative RT-PGR analysis. The experiments were repeated three times.

\section{Measurement of Drosophila Body Weight}

Eggs laid by five female flies for $6 \mathrm{~h}$ at $25^{\circ} \mathrm{C}$ were cultured to avoid over-crowding and lack of nutrition. For weight of individual fly, over 50 three day-old adult male flies were measured with the balancer (METTLER AJ100) and divided with the number of flies. At least three experiments were performed in each assay.

\section{Quantitative RT-PCR Analysis}

Adult heads from 20 flies were collected for RNA preparation. Total RNA was extracted using the easy-BLUE (TM) reagent (iNtRON biotechnology). All RNA samples were treated with RNase-free DNase (Promega). cDNA was synthesized using a SuperScript III First-Strand Synthesis System (Invitrogen). For quantitative RT-PCR analysis, ABI Prism 7900 Sequence Detection System (Applied Biosystems) and SyberGreen PCR Core reagents (Applied Biosystems) were used. mRNA levels were expressed as the relative fold change against the normalized $r p 49$ mRNA. The comparative cycle threshold (Ct) method (User Bulletin 2, Applied Biosystems) was used to analyze the data. All experiments were repeated at least three times. The statistical significance was tested by Microsoft Excel-based application for the student $t$-test statistical analysis. Primers used in the RT-PCR analyses were listed in Table S2.

\section{Generation of the Minibrain and sNPFR1 Antisera and Immunostaining in the Adult Brain of Drosophila}

Minibrain antiserum was generated by the immunization of rabbits with the synthetic peptide (CQHRVRNWPTNGNQ) corresponding to the N-terminal sequence (75-88) of the Minibrain-H protein. Antiserum against sNPFR1 was generated by the immunization of rat with the synthetic peptide (GEAIGAGGGAELGRRIN) corresponding to the C-terminal sequence
(585-600) of the sNPFRl protein. For immunostaining, adult brain from newly eclosed flies (3 day old) was dissected in PBS, fixed in $4 \%$ paraformaldehyde, and blocked in 5\% BSA and 5\% normal goat serum. Primary antibodies were incubated two days at $4^{\circ} \mathrm{C}$ and secondary antibodies were incubated for $2 \mathrm{~h}$ at room temperature. The tissues were mounted in the DABCO solution (70\% glycerol, 2.5\% DABCO, Sigma, St Louis, MO) and fluorescence images were acquired by FluoView confocal microscope (Olympus). sNPF (1:200), sNPFR1 (1:200), and Minibrain (1:200) primary antibodies, and anti-rat IgG Alexa 488, anti-rabbit IgG Alexa 488, or Alexa 594 (1:200, Molecular Probes) and antiguinea pig Cy5 (1:200, Jackson ImmunoResearch) secondary antibodies were used.

\section{Western Blot Analysis}

The cells were lysed by the Lysis buffer (Cell signaling) containing NaF, PMSF and $\mathrm{Na}_{3} \mathrm{VO}_{4}$. Total cell lysates were immunoprecipitated with Sirtl antibody (Cell signaling) and protein A-agarose (Pierce). The immunoprecipitates were washed three times with Lysis buffer and solubilized in the SDS sample buffer $(63.5 \mathrm{mM}$ Tris-HCl; pH 6.8, 2\% w/v SDS, 10\% glycerol, $50 \mathrm{mM}$ DTT, $0.01 \% \mathrm{w} / \mathrm{v}$ bromphenol blue). Western blot analyses were performed as described previously [2]. PhosphoCREB, phospho-Threonine, FoxO1 (1:1000, Cell signaling), AcFKHR (1:1000, Santa Cruze), $\beta$-actin (1:3000, Abcam) primary antibodies, and horseradish peroxidase-conjugated anti-rabbit IgG (1:5000, Santa Cruze) and anti-mouse IgG secondary antibody (1:5000, Sigma) were used.

\section{cAMP Assay}

Intracellular cAMP was measured with the cAMP Biotrak Enzyme Immunoassay Kit (GE Healthcare) by the manufacturer's instruction. Briefly, samples were incubated with anti-cAMP antibody, which was immobilized in the secondary antibody coated micro-plates. Following enzyme substrate conversion, an optical density was measured at $450 \mathrm{~nm}$ with microplate reader (Fluostar Optima, BMG labtech). cAMP concentration was expressed as the cAMP pM per mg of protein and converted to the fold change relative to the basal control value.

\section{ChIP-on-chip and ChIP-PCR Analysis}

About 250 of 3-day-old W[DAH] female flies were collected after $12 \mathrm{~h}$ starvation. Then, flies were homogenized and crosslinked in 1X PBS containing 1\% formaldehyde. The ChIP protocol was performed as described in Teleman et al. [56]. Immunoprecipitation was performed using Dynal protean G beads (Invitrogen) and anti-dFOXO antibody (a gift from Heather Broihier). Purified DNA was amplified and labeled following Affymetrix ChIP Assay Protocol. Drosophila Tiling 2.0R Array was used to detect dFOXO binding enrichment. For ChIP-PCR analysis, about $10^{8}$ of BG2-c6 cells were treated with sNPF2 peptide as described above. sNPF-treated and untreated cells were cross-linked with $1 \%$ formaldehyde. After immunoprecipitation with the CREB antibody (Cell signaling) and Protein A Sepharose CL-4B (GE Healthcare), quantitative RT-PCR analysis was performed using input DNA and immunoprecipitated DNA for the CREB binding site in the $m n b$ promoter region and the $3^{\text {rd }}$ axon of Actin5C.

\section{Statistics}

Values in the paper are presented as means \pm s.e.m. Statistical significant of all data were evaluated by the One-way ANOVA test 
(GraphPad Prism software). $P<0.05$ was accepted as statistically significant.

\section{Supporting Information}

Figure S1 (A) mnb mRNA expression levels of Figure 1A and UAS controls. (B) Relative food consumption of Figure $1 \mathrm{~B}$ and $U A S$ controls. (C) Relative food consumption of Figure $3 \mathrm{C}$, Figure 4E, and $U A S$ controls.

(TIF)

Figure S2 sNPFR1-Gal4 expression was detected by sNPFR1Gal4>UAS-DsRed (sNPFR1>DsRed) in the fly adult brain. (A-D) In the anterior focal planes, $s N P F R 1>D s R e d$ was detected in the optic lobes (OL, A), insulin producing cells (IPCs, C), mushroom body $(\mathrm{MB}, \mathrm{B})$, and subesophageal ganglions (SOG, D). (E, F) In the posterior focal planes, $s$ NPFR1 $>$ DsRed was detected in the median neurons above esophagus (E, dot box; F, asterisk). Scale bars are $100 \mu \mathrm{m}(\mathrm{A}, \mathrm{E}), 50 \mu \mathrm{m}(\mathrm{B}, \mathrm{D}, \mathrm{F})$ and $30 \mu \mathrm{m}(\mathrm{C})$.

(TIF)

Figure S3 (A) Western blots with the Mnb antibody in the sNPF-Gal4 control, $s \mathcal{N} P F$ overexpression $(s \mathcal{N} P F>2 x s \mathcal{N} P F)$, sNPF ${ }^{\text {c0448 }}$ mutant, sNPFR1-Gal4 control, sNPFR1 overexpression $(s \mathcal{N} F R 1>s \mathcal{N} P F R 1)$, and $s \mathcal{N} P F R 1$ inhibition $(s N P F R 1>s \mathcal{N} P F R 1-R i)$. (B-F) Numbers of strong Mnb expression neurons (asterisks) are similar in the sNPFR1-Gal4 control, sNPFR1 overexpression $(s \mathcal{N} F R 1>s \mathcal{N} P F R 1), \quad s \mathcal{N} P F R 1$ inhibition $\left(s \mathcal{N} P F R 1>{ }_{s} N P F R 1-R i\right)$, and $s \mathcal{N} P F^{c 00448}$ mutant. Scale bars are $100 \mu \mathrm{m}$.

(TIF)

Figure S4 (A) The mnb genomic organization. Open boxes represent exons, the triangle shows the p-element insertion site in $m n b^{G 1767}$, and an arrow indicates the transcriptional initiation of the $m n b H$ isoform containing the longest coding sequences among $m n b$ isoforms. mnb deletion mutants ( $m n b^{d 305}$ and $\left.m n b^{d 419}\right)$ were generated by imprecise excisions of the inserted p-element. (B) $m n b$ mRNA expression levels in the $m n b$ overexpression $(s \mathcal{N} F R 1>m n b)$, inhibition (sNPFR1>mnb-Ri), and $m n b^{G 1767}$ mutant. (C) Western blot with the Mnb antibody in the w- control and $m n b^{G 1767}$ mutant. (D) $m n b$ overexpression $(s \mathcal{N} P F R 1>m n b)$ increased the body weight compared with the $s \mathcal{N} P F R 1$-Gal4 control whereas mnb suppression (sNPFR1>mnb-Ri, mnb $b^{G 1767}$ ) decreased the body weight. (E) Amount of food intake by the normalized to body mass and to the number of flies. Data are presented as means \pm s.e.m. from three independent experiments. ${ }^{*} P<0.05$ (One-way ANOVA analysis).

(TIF)

Figure S5 Adult specific food intake assay using the tubGalo0ts inducible system. (A) In the $22^{\circ} \mathrm{C}$ permissive temperature condition in which tubGalo0ts suppress sNPFR1-Gal4 expression, mnb overexpression (sNPFR1-Gal4+tubGalo0ts>mnb, sNPFR1Gal4+tubGal80ts $>2 X m n b$ ) and mnb inhibition (sNPFR1-Gal4+tubGal80ts $>m n b-R i)$ flies did not change the amount of food intake compared with the control flies (sNPFR1-Gal4;tub-Galo0ts). (B) In the $30^{\circ} \mathrm{C}$ restrictive temperature in which tubGalo0ts cannot suppress sNPFR1-Gal4, the mnb overexpression increased food intake compared with the control and the mnb inhibition

\section{References}

1. Morton GJ, Cummings DE, Baskin DG, Barsh GS, Schwartz MW (2006) Central nervous system control of food intake and body weight. Nature 443: 289-295.

2. Lee KS, You KH, Choo JK, Han YM, Yu K (2004) Drosophila short neuropeptide F regulates food intake and body size. J Biol Chem 279: 5078150789. suppressed food intake. Data are presented as means \pm s.e.m. from three independent experiments. ${ }^{*} P<0.05$ (One-way ANOVA analysis).

(TIF)

Figure S6 (A) Expression levels of $s \mathcal{N} P F$ and $s \mathcal{N} P F R 1$ in Drosophila BG2-C6 cells after sNPF treatment. (B) cAMP level in Drosophila BG2-c6 cells after sNPF treatment. (C) cAMP level in mouse GT17 cells after NPY treatment.

(TIF)

Figure S7 Levels of mnb mRNA expression (A), relative food consumption (B), and $s \mathcal{N} P F$ mRNA expression $(\mathrm{C})$ in Gal4 and $U A S$ controls used in this study.

(TIF)

Figure S8 Promoter analysis of $m n b$ genes from twelve Drosophila species reveals that the cAMP-response element (CRE), which is TGACGTCA, was conserved in Drosophila species including $D$. melanogaster (Adapted and modified from UCSG Genome Browser at http://genome.ucsc.edu).

(TIF)

Figure S9 (A, B) RT-PCR analysis of sNPFR1, mnb, Sir2, and dFOXO mRNA in the sNPFR1-Gal4, sNPFR1>sNPFR1, $s \mathcal{N P F R} 1>m n b, s \mathcal{N} P F R 1>\operatorname{Sir} 2$, and $s \mathcal{N} P F 1>d F O X O$ overexpression and in the sNPFR1>sNPFR1-Ri, sNPFR1>mnb-Ri, sNPFR1>Sir2$R i$, and $s N P F R 1>d F O X O-R i$ inhibition. (C) sNPFR1 expression during fasting. (D) sNPFR1 mRNA expression was not changed in Dilp2 $>$ Dilp2 compared to the Dilp2-Gal4 control and in $s \mathcal{N P F R} 1>\operatorname{InR}$ and $s \mathcal{N} P F R 1>\operatorname{InR}{ }^{D \mathcal{N}}$ compared to the $s \mathcal{N} P F R 1-$ Gal4 control.

(TIF)

Figure S10 Promoter analysis of $s \mathcal{N P F}$ genes from twelve Drosophila species reveals that the $\mathrm{dFOXO}$ binding site, which is RWWAACA, was conserved in five Drosophila species including $D$. melanogaster (Adapted and modified from UCSG Genome Browser at http://genome.ucsc.edu).

(TIF)

Figure S11 hDyrk1a transgenic mice showed slightly increased body weight. Data are presented as means \pm s.e.m. ${ }^{*} P<0.05$.

(TIF)

Table S1 mnb expression in the DNA microarray analysis. (DOC)

Table S2 PCR primer sequences in this study. (DOC)

\section{Acknowledgments}

We thank J. S. Lee for helpful comments on the manuscript and K. H. Jeong for technical support.

\section{Author Contributions}

Conceived and designed the experiments: S-HH K-SL S-JK MT KY. Performed the experiments: S-HH K-SL S-JK A-KK HB M-SJ. Analyzed the data: S-HH K-SL S-JK O-YK W-JS KY. Wrote the paper: S-HH KSL MT KY.

3. Lee KS, Kwon OY, Lee JH, Kwon K, Min KJ, et al. (2008) Drosophila short neuropeptide $\mathrm{F}$ signalling regulates growth by ERK-mediated insulin signalling. Nat Cell Biol 10: 468-475.

4. Guimera J, Casas C, Estivill X, Pritchard M (1999) Human minibrain homologue (MNBH/DYRK1): characterization, alternative splicing, differential tissue expression, and overexpression in Down syndrome. Genomics 57: 407-418. 
5. Guimera J, Casas C, Pucharcos C, Solans A, Domenech A, et al. (1996) A human homologue of Drosophila minibrain (MNB) is expressed in the neuronal regions affected in Down syndrome and maps to the critical region. Hum Mol Genet 5: 1305-1310.

6. Tejedor F, Zhu XR, Kaltenbach E, Ackermann A, Baumann A, et al. (1995) minibrain: a new protein kinase family involved in postembryonic neurogenesis in Drosophila. Neuron 14: 287-301.

7. Song WJ, Sternberg LR, Kasten-Sportes C, Keuren ML, Chung SH, et al. (1996) Isolation of human and murine homologues of the Drosophila minibrain gene: human homologue maps to $21 \mathrm{q} 22.2$ in the Down syndrome "critical region". Genomics 38: 331-339.

8. Dowjat WK, Adayev T, Kuchna I, Nowicki K, Palminiello S, et al. (2007) Trisomy-driven overexpression of DYRK1A kinase in the brain of subjects with Down syndrome. Neurosci Lett 413: 77-81.

9. Fotaki V, Dierssen M, Alcantara S, Martinez S, Marti E, et al. (2002) Dyrk1A haploinsufficiency affects viability and causes developmental delay and abnormal brain morphology in mice. Mol Cell Biol 22: 6636-6647.

10. Moller RS, Kubart S, Hoeltzenbein M, Heye B, Vogel I, et al. (2008) Truncation of the Down syndrome candidate gene DYRK1A in two unrelated patients with microcephaly. Am J Hum Genet 82: 1165-1170.

11. van Bon BW, Hoischen A, Hehir-Kwa J, de Brouwer AP, Ruivenkamp C, et al. (2011) Intragenic deletion in DYRK1A leads to mental retardation and primary microcephaly. Clinical Genetics 79: 296-299.

12. Kitamura T, Feng Y, Kitamura YI, Chua SC, Jr., Xu AW, et al. (2006) Forkhead protein FoxOl mediates Agrp-dependent effects of leptin on food intake. Nat Med 12: 534-540.

13. Ramadori G, Lee CE, Bookout AL, Lee S, Williams KW, et al. (2008) Brain SIRT1: anatomical distribution and regulation by energy availability. J Neurosci 28: 9989-9996.

14. Dietrich MO, Antunes G, Geliang G, Liu ZW, Borok E, et al. (2010) Agrp neurons mediate Sirtl's action on the melanocortin system and energy balance: roles for Sirtl in neuronal firing and synaptic plasticity. J Neurosci 30: 1181511825.

15. Cakir I, Perello M, Lansari O, Messier NJ, Vaslet CA, et al. (2009) Hypothalamic Sirtl regulates food intake in a rodent model system. PLoS ONE 4: e8322. doi:10.1371/journal.pone.0008322

16. Lee KS, Hong SH, Kim AK, Ju SK, Kwon OY, et al. (2009) Processed short neuropeptide $\mathrm{F}$ peptides regulate growth through the ERK-insulin pathway in Drosophila melanogaster. FEBS Lett 583: 2573-2577.

17. Ja WW, Carvalho GB, Mak EM, de la Rosa NN, Fang AY, et al. (2007) Prandiology of Drosophila and the CAFE assay. Proc Natl Acad Sci U S A 104: 8253-8256.

18. McGuire SE, Mao Z, Davis RL (2004) Spatiotemporal gene expression targeting with the TARGET and gene-switch systems in Drosophila. Sci STKE 2004: pl6.

19. Ui K, Nishihara S, Sakuma M, Togashi S, Ueda R, et al. (1994) Newly established cell lines from Drosophila larval CNS express neural specific characteristics. In Vitro Cellular and Developmental Biology Animal 30A: 209216.

20. Ritter SL, Hall RA (2009) Fine-tuning of GPCR activity by receptor-interacting proteins. Nat Rev Mol Cell Biol 10: 819-830.

21. Mayr B, Montminy M (2001) Transcriptional regulation by the phosphorylation-dependent factor CREB. Nat Rev Mol Cell Biol 2: 599-609.

22. Mellon PL, Windle JJ, Goldsmith PC, Padula CA, Roberts JL, et al. (1990) Immortalization of hypothalamic GnRH neurons by genetically targeted tumorigenesis. Neuron 5: 1-10.

23. Blomqvist AG, Herzog H (1997) Y-receptor subtypes-how many more? Trends Neurosci 20: 294-298.

24. Kamiji MM, Inui A (2007) Neuropeptide y receptor selective ligands in the treatment of obesity. Endocr Rev 28: 664-684.

25. Zhang X, Odom DT, Koo SH, Conkright MD, Canettieri G, et al. (2005) Genome-wide analysis of cAMP-response element binding protein occupancy, phosphorylation, and target gene activation in human tissues. Proc Natl Acad Sci U S A 102: 4459-4464.

26. Guo X, Williams JG, Schug TT, Li X (2010) DYRK1A and DYRK3 promote cell survival through phosphorylation and activation of SIRT1. J Biol Chem 285: 13223-13232.

27. Kim MS, Pak YK, Jang PG, Namkoong C, Choi YS, et al. (2006) Role of hypothalamic Foxol in the regulation of food intake and energy homeostasis. Nat Neurosci 9: 901-906.

28. Wu Q Zhang Y, Xu J, Shen P (2005) Regulation of hunger-driven behaviors by neural ribosomal S6 kinase in Drosophila. Proc Natl Acad Sci U S A 102: 13289-13294

29. Ikeya T, Galic M, Belawat P, Nairz K, Hafen E (2002) Nutrient-dependent expression of insulin-like peptides from neuroendocrine cells in the CNS contributes to growth regulation in Drosophila. Current Biology 12: 1293-1300.
30. Huang H, Tindall DJ (2007) Dynamic FoxO transcription factors. J Cell Sci 120: 2479-2487.

31. Puig O, Mattila J (2011) Understanding Forkhead box class $\mathrm{O}$ function: lessons from Drosophila melanogaster. Antioxid Redox Signal 14: 635-647.

32. Brunet A, Bonni A, Zigmond MJ, Lin MZ, Juo P, et al. (1999) Akt promotes cell survival by phosphorylating and inhibiting a Forkhead transcription factor. Cell 96: 857-868.

33. Nielsen MD, Luo X, Biteau B, Syverson K, Jasper H (2008) 14-3-3 Epsilon antagonizes FoxO to control growth, apoptosis and longevity in Drosophila. Aging Cell 7: 688-699.

34. Matsuzaki H, Daitoku H, Hatta M, Aoyama H, Yoshimochi K, et al. (2005) Acetylation of Foxol alters its DNA-binding ability and sensitivity to phosphorylation. Proc Natl Acad Sci U S A 102: 11278-11283.

35. ParkJ, Song WJ, Chung KC (2009) Function and regulation of Dyrk1A: towards understanding Down syndrome. Cell Mol Life Sci 66: 3235-3240.

36. Korenberg JR, Chen XN, Schipper R, Sun Z, Gonsky R, et al. (1994) Down syndrome phenotypes: the consequences of chromosomal imbalance. Proc Natl Acad Sci U S A 91: 4997-5001.

37. Magge SN, O’Neill KL, Shults J, Stallings VA, Stettler N (2008) Leptin levels among prepubertal children with Down syndrome compared with their siblings. J Pediatr 152: 321-326.

38. Melville CA, Cooper SA, McGrother CW, Thorp CF, Collacott R (2005) Obesity in adults with Down syndrome: a case-control study. J Intellect Disabil Res 49: 125-133.

39. Obici S, Feng Z, Karkanias G, Baskin DG, Rossetti L (2002) Decreasing hypothalamic insulin receptors causes hyperphagia and insulin resistance in rats. Nat Neurosci 5: 566-572.

40. Sipols AJ, Baskin DG, Schwartz MW (1995) Effect of intracerebroventricular insulin infusion on diabetic hyperphagia and hypothalamic neuropeptide gene expression. Diabetes 44: 147-151.

41. Varghese J, Lim SF, Cohen SM (2010) Drosophila miR-14 regulates insulin production and metabolism through its target, sugarbabe. Genes Dev 24: 27482753.

42. Rulifson EJ, Kim SK, Nusse R (2002) Ablation of insulin-producing neurons in flies: growth and diabetic phenotypes. Science 296: 1118-1120.

43. Min KJ, Yamamoto R, Buch S, Pankratz M, Tatar M (2008) Drosophila lifespan control by dietary restriction independent of insulin-like signaling. Aging Cell 7: 199-206.

44. Gronke S, Clarke DF, Broughton S, Andrews TD, Partridge L (2010) Molecular evolution and functional characterization of Drosophila insulin-like peptides. PLoS Genet 6: e1000857. doi:10.1371/journal.pgen.1000857

45. McLeod CJ, Wang L, Wong C, Jones DL (2010) Stem cell dynamics in response to nutrient availability. Current Biology 20: 2100-2105.

46. Root CM, Ko KI, Jafari A, Wang JW (2011) Presynaptic facilitation by neuropeptide signaling mediates odor-driven food search. Cell 145: 133-144.

47. Rubin GM, Spradling AC (1982) Genetic transformation of Drosophila with transposable element vectors. Science 218: 348-353.

48. Griswold AJ, Chang KT, Runko AP, Knight MA, Min KT (2008) Sir2 mediates apoptosis through JNK-dependent pathways in Drosophila. Proc Natl Acad Sci U S A 105: 8673-8678.

49. Iijima-Ando K, Wu P, Drier EA, Iijima K, Yin JC (2005) cAMP-response element-binding protein and heat-shock protein 70 additively suppress polyglutamine-mediated toxicity in Drosophila. Proc Natl Acad Sci U S A 102: 10261-10266.

50. Ohlmeyer JT, Kalderon D (1997) Dual pathways for induction of wingless expression by protein kinase A and Hedgehog in Drosophila embryos. Genes Dev 11: 2250-2258.

51. Ueno K, Kohatsu S, Clay C, Forte M, Isono K, et al. (2006) Gsalpha is involved in sugar perception in Drosophila melanogaster. J Neurosci 26: 6143-6152.

52. Brand AH, Perrimon N (1993) Targeted gene expression as a means of altering cell fates and generating dominant phenotypes. Development 118: 401-415.

53. Xu K, Zheng X, Sehgal A (2008) Regulation of feeding and metabolism by neuronal and peripheral clocks in Drosophila. Cell Metab 8: 289-300.

54. Wong R, Piper MD, Blanc E, Partridge L (2008) Pitfalls of measuring feeding rate in the fruit fly Drosophila melanogaster. Nat Methods 5: 214-215; author reply 215

55. Ahn KJ, Jeong HK, Choi HS, Ryoo SR, Kim YJ, et al. (2006) DYRK1A BAC transgenic mice show altered synaptic plasticity with learning and memory defects. Neurobiol Dis 22: 463-472.

56. Teleman AA, Hietakangas V, Sayadian AC, Cohen SM (2008) Nutritional control of protein biosynthetic capacity by insulin via Myc in Drosophila. Cell Metab 7: 21-32. 